\title{
The elusive gluon
}

\author{
Mikael Chala, ${ }^{a, b}$ José Juknevich, ${ }^{c, d}$ Gilad Perez $^{e}$ and José Santiago ${ }^{a, f}$ \\ ${ }^{a}$ CAFPE and Departamento de Física Teórica y del Cosmos, \\ Universidad de Granada, E-18071 Granada, Spain \\ ${ }^{b} D E S Y$, \\ Notkestrasse 85, 22607 Hamburg, Germany \\ ${ }^{c} S I S S A / I S A S$, \\ I-34136 Trieste, Italy \\ ${ }^{d}$ INFN - Sezione di Trieste, \\ 34151 Trieste, Italy \\ ${ }^{e}$ Department of Particle Physics and Astrophysics, Weizmann Institute of Science, \\ Rehovot 76100, Israel \\ ${ }^{f}$ CERN, Theory Division, \\ CH-1211 Geneva 23, Switzerland \\ E-mail: mikael.chala@desy.de, jose.juknevich@sissa.it, \\ gilad.perez@weizmann.ac.il, jsantiago@ugr.es
}

ABSTRACT: We study the phenomenology of vector resonances in the context of natural composite Higgs models. A mild hierarchy between the fermionic partners and the vector resonances can be expected in these models based on the following arguments. Both direct and indirect (electroweak and flavor precision) constraints on fermionic partners are milder than the ones on spin one resonances. Also the naturalness pressure coming from the top partners is stronger than that induced by the gauge partners. This observation implies that the search strategy for vector resonances at the LHC needs to be modified. In particular, we point out the importance of heavy gluon decays (or other vector resonances) to top partner pairs that were overlooked in previous experimental searches at the LHC. These searches focused on simplified benchmark models in which the only new particle beyond the Standard Model was the heavy gluon. It turns out that, when kinematically allowed, such heavy-heavy decays make the heavy gluon elusive, and the bounds on its mass can be up to $2 \mathrm{TeV}$ milder than in the simpler models considered so far for the LHC14. We discuss the origin of this difference and prospects for dedicated searches.

Keywords: Beyond Standard Model, Technicolor and Composite Models, Field Theories in Higher Dimensions

ARXIV EPRINT: 1411.1771 


\section{Contents}

1 Introduction 1

2 The model 3

3 LHC signatures $\quad 6$

4 Current bounds $\quad 8$

5 Method 10

$\begin{array}{ll}5.1 \text { Event generation } & 10\end{array}$

5.2 Analysis details 11

$\begin{array}{lll}5.3 & \text { Statistical procedure } & 13\end{array}$

$\begin{array}{ll}\text { 5.4 Comparison with ATLAS benchmark analysis } & 14\end{array}$

6 Results 14

$\begin{array}{lll}\text { 6.1 Physical distributions } & 16\end{array}$

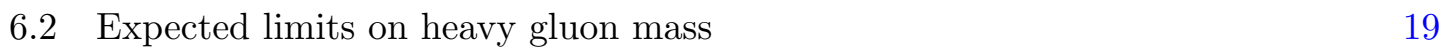

$\begin{array}{ll}6.3 \text { Recovering the signal } & 21\end{array}$

7 Conclusions and outlook $\quad 23$

$\begin{array}{ll}\text { A Model description } & 25\end{array}$

\section{Introduction}

The LHC discovery of the Higgs boson, with a light mass and couplings consistent with the Standard Model (SM) prediction, reinforces the fine tuning problem. In the SM the Higgs mass is radiatively unstable and it has uncontrolled sensitivity to microscopic dynamics. A simple possibility to stabilize the Higgs mass and the electroweak scale in a controlled manner is to add new fields to the SM, with the same gauge quantum numbers as the SM fields, such that the contributions of the new fields to the Higgs mass eliminate the UV sensitivity. The most severe known sensitivity of the Higgs to quantum corrections arises as a result of its large coupling to the top quark. To ensure the stabilization of the electroweak scale, the virtual contributions of some of the new particles to the Higgs mass should cancel the contributions coming from the SM top quarks. These new states are collectively denoted as top partners. In known examples, the partners might be scalars as in the case of supersymmetry or fermions as in the case of composite Higgs models (CHMs). Naturalness also requires the presence of additional states, partners of the electroweak gauge bosons, fermions in the supersymmetric case and massive vectors in CHMs. 
Our focus in this paper is to study the interplay between the collider phenomenology of the massive vectors and the top partners within a class of natural CHMs. As we shall see below, the search strategy that was chosen so far by the LHC experiments regarding the vector resonance might be incomplete and can potentially be improved in an essential manner. Our claim stems from the following three observations regarding the status of natural composite Higgs models (see also [1]):

- The direct constraints on the mass of the top partners are weaker than those of the vector resonances. The lower bound on the mass of composite-Higgs fermionic states is roughly $800 \mathrm{GeV}$ (see e.g. refs. [2, 3] for recent results), while the corresponding lower bounds on the mass of a colour-octet spin-one resonance mass is in the $2-2.5 \mathrm{TeV}$ region (see e.g. refs. $[4,5]$ ).

- The indirect constraints on the mass of the top partners are weaker than those of the vector resonances. The lower bounds from electroweak (EW) precision tests (see [68]) and flavor physics [9] on the fermion partners are roughly of a $\mathrm{TeV}$, while the corresponding lower bounds on the vector resonance masses is in the multi- $\mathrm{TeV}$ range.

- The naturalness pressure on the top partners is stronger than that of the vector resonances, as is well known (see e.g. [10] and refs. therein). In a natural theory the top partners are required to lie below the $\mathrm{TeV}$ scale whereas the vector resonances can have masses beyond the TeV scale. Furthermore, the combination of LEP and Tevatron data constrains the model's decay constant $f$ to lie above the $f>\mathcal{O}(800 \mathrm{GeV})$ scale $[8,11]$. Therefore the composite fermion resonances would be somewhat heavier with masses probably larger than $f$. Thus, requiring similar level of tuning on the vector resonances will send their masses to the multi-TeV range. The measured value of the Higgs mass further increases this pressure as pointed out in $[12-17]$ (see $[18,19]$ for a discussion of effects that could partially alleviate this pressure).

Adding the information in these items leads to a potentially viable spectrum of natural pseudo Nambu-Goldstone Boson (pNGB) composite models where the top partners are relatively light, with masses around the $\mathrm{TeV}$ scale, while the spin one states such as the $Z^{\prime} / W^{\prime}$ and the colour octet resonances or the Kaluza-Klein (KK) excitations in warped extra-dimensional Randall-Sundrum (RS) models [20,21] are expected to have masses in the multi-TeV region. This mild hierarchy between the masses of the vector resonances and the fermionic partners not only improves the consistency of the framework but it also suggests a qualitative change in the current search strategies for the composite vector resonances. The reason is simple: in the above set up we generically expect that the inter-composite couplings, or the couplings between the resonances, would dominate over the couplings between the resonance and the SM fields (that cannot be all composite due to various constraints). Thus, unlike in the original theoretical constructions, the mild hierarchy in scales implies that the resonances such as the KK gluon [22, 23] and their EW counterparts [24] (including the celebrated $Z^{\prime}$ ) would preferably decay to pairs of top partners instead of pairs of SM fields, such as tops, the final state that has been most frequently analysed thus far. 
We study in this paper in a quantitative way the implications that top partners have on heavy gluon searches (see [25] for a related analysis of $W^{\prime}$ resonances). The complementary effect, namely the implications that heavy gluon resonances have on top partner searches was discussed in detail in the context of holographic composite Higgs models in [26]. We will show that the larger width and the large number of new channels, typically involving $t \bar{t}+X$ in the final state with $X$ a pair of SM gauge or Higgs bosons, make the heavy gluon much more elusive than the one in which top partners are absent. Current bounds can be easily a few hundred $\mathrm{GeV}$ less stringent for realistic heavy gluons than the ones currently being reported. At the LHC14 this difference can go up to almost $2 \mathrm{TeV}$ (see also $[27,28]$ ). We discuss the interplay of resolved and boosted analyses in these searches and we finally propose simple extensions of current analyses that would allow one to recover a good fraction of the lost sensitivity. The emphasis will be in the natural region of parameter space in which pair production of top partners is kinematically open. The case of heavier top partners for which single production in association with a SM (top or bottom) quark is the dominant channel has been studied in detail in [29-33] and will be used here just for comparison purposes.

The rest of the article is organized as follows. In section 2 we describe the main features of our minimal CHM. In section 3 we discuss the main implications of top partners on the phenomenology of the heavy gluon. In sections 4 and 5 we summarize the existing experimental bounds on heavy gluons and describe in detail our reconstruction method. Our main results, the current and predicted bounds for the LHC14 on the heavy gluon in the presence of top partners are reported in section 6, where we also present our proposed search strategies to improve the sensitivity to the heavy gluon. Finally we conclude in section 7. A detailed description of the model is provided in the appendix.

\section{The model}

The general discussion in the previous section shows that, quite generically, the phenomenology of the heavy gluon in realistic CHMs is likely to be very different from the one in the models currently being used to interpret experimental searches. In order to make a quantitative estimate of the effects of such differences and their implications for LHC searches, we consider the Minimal Composite Higgs model, based on the $\mathrm{SO}(5) / \mathrm{SO}(4)$ coset, with composite fermions transforming in the vector representation (5) of $\mathrm{SO}(5)[34,35]$. This is the minimal model that contains only the Higgs doublet as the pNGB of the symmetry breaking and incorporates custodial symmetry to protect the $T$ parameter and the $Z b_{L} \bar{b}_{L}$ coupling [36]. For the sake of simplicity we are going to consider a simplified version of this model, developed in [37], in which the right-handed $(\mathrm{RH})$ top quark is fully composite and only the first level of fermion resonances is included. The model is denoted $\mathrm{MCH}_{5}$, where the 5 indicates the $\mathrm{SO}(5)$ representation of the composite operator that mixes with the SM left-handed quark doublets realizing the partial compositeness scenario, and the 4 stands for the $\mathrm{SO}(4)$ representation of the lightest fermion 
resonances which read explicitly

$$
\Psi=\frac{1}{\sqrt{2}}\left(\begin{array}{c}
i B-i X_{5 / 3} \\
B+X_{5 / 3} \\
i T+i X_{2 / 3} \\
-T+X_{2 / 3}
\end{array}\right) .
$$

In terms of $\mathrm{SU}(2)_{L} \times \mathrm{U}(1)_{Y}$ representations, the fourplet $\Psi$ gives rise to two doublets. One doublet $(T, B)$ with hypercharge $1 / 6$, as the SM left-handed doublet, and a second doublet $\left(X_{5 / 3}, X_{2 / 3}\right)$ with hypercharge $7 / 6$, containing an exotic state with charge $5 / 3, X_{5 / 3}$, and a charge $2 / 3$ state, $X_{2 / 3}$. After electroweak symmetry breaking there is a linear combination of the two charge $2 / 3$ quarks, denoted $X_{2 / 3}^{\prime}$, that is degenerate with $X_{5 / 3}$ whereas the orthogonal combination, denoted $T^{\prime}$, and $B$ are somewhat heavier and with a small mass splitting between them. They decay almost in all the parameter space into a top quark and a SM gauge or Higgs boson, with approximately equal branching ratio $(B R)$ into all open channels

$$
\begin{aligned}
B R\left(X_{5 / 3} \rightarrow t W^{+}\right) & =B R\left(B \rightarrow t W^{-}\right)=1, \\
B R\left(X_{2 / 3}^{\prime} \rightarrow t Z\right) & \approx B R\left(X_{2 / 3}^{\prime} \rightarrow t H\right) \approx B R\left(T^{\prime} \rightarrow t Z\right) \approx B R\left(T^{\prime} \rightarrow t H\right) \approx \frac{1}{2} .
\end{aligned}
$$

Apart from the scale characterizing the strong coupling, that we fix to $f=800 \mathrm{GeV}$, and the mass of the degenerate fermion resonances, $M_{\Psi}=M_{X_{2 / 3}^{\prime}}=M_{X_{5 / 3}}$, there are only three order one dimensionless parameters in the original model. One of these parameters is fixed by the top mass (we take it to be $y$ in the notation of ref. [37], see the appendix for details) and the other two have a small effect on the phenomenology that we are investigating so we just fix them to $c_{1}=0.7$ and $c_{2}=1.7$, again in the notation of ref. [37].

Regarding the heavy gluon we asume that there is a composite heavy vector color octet that couples to the composite quarks (including $t_{R}$ ) with a coupling $g_{c}$ and an elementary massless color octet that couples with the elementary fields with a coupling $g_{e}$. The two color-octet vectors mix linearly in such a way that a linear combination remains massless, the partially composite SM gluon, with a coupling

$$
g_{s}=g_{e} \cos \theta_{3}=g_{c} \sin \theta_{3} .
$$

The orthogonal combination is the heavy gluon we are interested in. The coupling of elementary and composite fermions to the heavy gluon read

$$
\begin{aligned}
G \bar{\psi}_{\text {elem }} \psi_{\text {elem }} & :-\frac{g_{s}^{2}}{\sqrt{g_{c}^{2}-g_{s}^{2}}}, \\
G \bar{\psi}_{\text {comp }} \psi_{\text {comp }} & : \sqrt{g_{c}^{2}-g_{s}^{2}} .
\end{aligned}
$$

Once we go to the physical basis for the fermions, they become partially composite and their couplings depend on the degree of compositeness. For simplicity we consider that all first two generation quarks, together with the $\mathrm{RH}$ component of the bottom quark, are purely elementary. The heavy gluon brings two new parameters in the game, the composite 
coupling $g_{c}$ and the heavy gluon mass $M_{G}$. The mass will be taken as a free parameter that we scan over while we fix the composite coupling to $g_{c}=4$ in our analyses. This is a somewhat smaller value of the one that would correspond to the original RS model, which would lead to too large a heavy gluon width when decays into top partners are kinematically open. In practice this means that the coupling of the heavy gluon to mostly composite states is smaller and the one to mostly elementary fields is larger than in the original RS model.

To summarize, we fix the following values of the parameters:

$$
g_{c}=4, \quad f=800 \mathrm{GeV}, \quad c_{1}=0.7, \quad c_{2}=1.7 .
$$

This choice of $g_{c}$ impies a coupling of the heavy gluon to the light SM quarks $g_{G q q}=-0.377$. We take two benchmark values for the top partner mass parameter $M_{\Psi}$ :

$$
\begin{array}{ll}
M_{\Psi}=M_{G} & (\text { noTP }), \\
M_{\Psi}=1 \mathrm{TeV} & \text { (lightTP). }
\end{array}
$$

In the first model, that we call not top partners (noTP), the decay into top partners, either singly or in pairs, is not kinematically allowed and therefore this model reproduces the main features of the model that is currently used to interpret LHC searches. The second model, called light top partners (lightTP), is the benchmark model for a realistic CHM, in which top partners are expected to be relatively light and therefore the decay into top partner pairs, or in association with a SM top or bottom, is kinematically allowed. Our choice of $1 \mathrm{TeV}$ for the top-partner mass is a compromise between what one would expect from the observed value of the Higgs mass and the current limits on top partners. The other two top partners are almost degenerate

$$
M_{T^{\prime}} \approx M_{B} \approx 1.13 \mathrm{TeV}
$$

This model has all the features we discussed in the introduction: a large gluon width, a small decay fraction into $t \bar{t}$ and a large one into $t \bar{t} X$-where $X$ stands for two SM gauge or Higgs bosons-. In order to disentangle the different effects we will also consider other three benchmark models in which we artificially modify some of the couplings to highlight some of the relevant features. These models are denoted by

$$
\begin{array}{lll}
\text { lightTP } & M_{\Psi}=1 \mathrm{TeV}, & \Gamma=\Gamma_{\text {noTP }}, \\
\text { lightTP }_{\text {notop }} & M_{\Psi}=1 \mathrm{TeV}, & g_{G t t}=0, \\
\text { singleTP }_{\text {narrow }} & M_{\Psi}=M_{G} / 2, & g_{G t t}=g_{G b_{L} b_{L}}=0,
\end{array}
$$

In all three cases we have rescaled the couplings of the composite (and $t_{L}, b_{L}$ ) quarks to have a narrow resonance (explicitly we have fixed the width to the one in the noTP model). Thus, the large width effect is removed in these models. The lightTP $\mathrm{Parrow}_{\text {model }}$ is a narrow-resonance version of lightTP, with quite similar decay patterns. In our second benchmark model, lightTP $\mathrm{P}_{\text {notop }}$, we have further set to zero the couplings to the top (and re-scaled again the couplings to the top partners to keep the same width) to ensure decay 


\begin{tabular}{|c|c|c|c|c|c|}
\hline Model & $\mathrm{BR}(G \rightarrow t t)$ & $\mathrm{BR}(G \rightarrow b b)$ & $\operatorname{BR}(G \rightarrow \Psi \Psi)$ & $\mathrm{BR}(G \rightarrow \Psi \psi)$ & $\Gamma_{G} / M_{G}$ \\
\hline noTP & 0.92 & 0.01 & 0 & 0 & 0.1 \\
\hline lightTP & 0.15 & 0.004 & 0.75 & 0.08 & 0.65 \\
\hline lightTP $\mathrm{Tarrow}$ & 0.14 & 0.01 & 0.7 & 0.08 & 0.1 \\
\hline lightTP notop & 0 & 0.01 & 0.82 & 0.09 & 0.1 \\
\hline singleTP ${ }_{\text {narrow }}$ & 0 & 0.007 & 0 & 0.94 & 0.14 \\
\hline
\end{tabular}

Table 1. Relevant heavy gluon parameters in our benchmark models for $M_{G}=2.5 \mathrm{TeV}$.

only to top partners. Finally, in the singleTP ${ }_{\text {narrow }}$ model we have chosen the top partner mass to favour single top partner production. Since the decay into pairs of top partners is kinematically forbidden, the width is relatively small. However, the fact that the RH top is fully composite leaves a small $B R$ into a top partner and a top or bottom quark. In order to increase the $B R$ into these channels we set the couplings to the top and left-handed bottom to zero and re-scale the couplings to $t T$ and $b B$ to keep the original width. As an illustration we provide in table 1 the values of the decay branching fractions and the width of the heavy gluon to the different fields for a reference mass $M_{G}=2.5 \mathrm{TeV}$.

\section{LHC signatures}

All the models analyzed in this work have one thing in common - when kinematically allowed, the heavy gluon has a large decay rate into pairs of top partners or a top partner and a top or bottom quark. We show as an example the branching ratios for heavy gluon decays in figure 1 as a function of $M_{G}$ for the lightTP model. Moreover, the total width of $G$ turns out to be generically in the range of $50 \%$ to $80 \%$ of the $G$ mass, which makes it a broad resonance and extremely challenging to discover. As stressed above, this is in sharp contrast with previous studies of heavy gluon searches at the LHC in which $G$ was assumed to be not too broad and to decay predominantly to $t \bar{t}$.

Here, we are going to show the main phenomenological differences between the heavy gluon in the $\mathrm{MCH}_{5}$ scenario and the heavy gluon in the RS KK gluon scenario in regard to final states from their decays and their kinematical reconstruction. We base our discussion on the class of models described in section 2. In order to disentangle the main effects that can make the heavy gluon elusive, in this section we focus on the results at partonic level and postpone a discussion on the full reconstruction of the heavy gluon resonance until section 6. This will give us a good handle on how well the LHC $t \bar{t}$ resonance searches, designed for RS KK gluon model in mind, will do at covering the much more general $\mathrm{MCH}_{5}$ parameter space. The parton-level analysis also gives us some useful information that is less sensitive to a particular experiment or reconstruction method.

Starting from the Lagrangian, described in detail in the appendix (see eq. (A.2)), one can derive the phenomenology of the heavy gluon. Since the coupling of the heavy gluon to ordinary gluons vanishes at tree level, the main rate of production at the LHC comes from the Drell-Yan process $q \bar{q} \rightarrow G$. The differential cross section as a function of $m_{\Psi \bar{\Psi}}^{\text {true }}$ at 


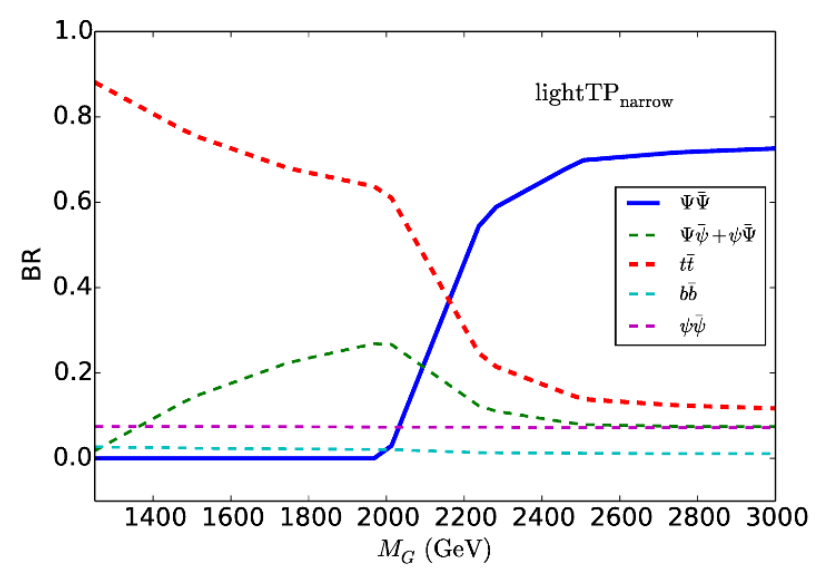

Figure 1. The heavy gluon branching ratios in the lightTP model for all channels. The blue solid line corresponds to the $\Psi \bar{\Psi}$ final state, with $\Psi=T^{\prime}, B, X_{2 / 3}^{\prime}, X_{5 / 3}$; the red dashed line to the $t \bar{t}$; the green dashed line to the $T^{\prime} \bar{t}+t \bar{T}^{\prime}$ and $B \bar{b}+b \bar{B}$; the cyan and purple dashed line correspond, respectively, to $b \bar{b}$ and light quarks.

LO is presented in figure 2 for $M_{G}=2.5 \mathrm{TeV}$ produced at the LHC with $\sqrt{s}=8 \mathrm{TeV}$ (left panel) and $\sqrt{s}=14 \mathrm{TeV}$ (right panel). The invariant mass for the heavy gluon in the noTP model is shown for comparison in the same figure. The invariant mass is calculated from the decay products of $G$ using truth level particles directly from Monte Carlo. The shape of the distribution for the noTP model simply corresponds to the Breit-Wigner factor convoluted with the corresponding parton distribution functions (PDF). By contrast, the invariant heavy gluon mass for the lightTP model is in average quite low and spread out. This is a direct consequence of the large width of the heavy gluon together with enhancement of PDF's at low $\mathrm{x}$, which lead to significant departure from the narrow-width approximation. In this case, a search for the heavy gluon would be quite a challenge, if not impossible. The invariant mass distributions of the narrow models, lightTP $\mathrm{Parrow}_{\text {and }}$ singleTP $\mathrm{Tarrow}_{\text {, }}$ are more sharply peaked around the heavy gluon mass and the distributions are more symmetric. The relatively small width of the heavy gluon for the narrow-resonance models suggests a new promising strategy for discovering the $G$, by searching for resonances in the invariant mass distribution of top partner pairs.

Considering the pattern of top partner decays, we can identify the following final channels:

$$
\begin{aligned}
& p p \rightarrow G \rightarrow X_{5 / 3} \bar{X}_{5 / 3} \rightarrow\left(t W^{+}\right)\left(\bar{t} W^{-}\right) \\
& p p \rightarrow G \rightarrow X_{2 / 3} \bar{X}_{2 / 3} \rightarrow(t Z+t h)(\bar{t} Z+\bar{t} h) \\
& p p \rightarrow G \rightarrow T \bar{T} \rightarrow\left(t Z+t h+b W^{+}\right)\left(\bar{t} Z+\bar{t} h+\bar{b} W^{-}\right) \\
& p p \rightarrow G \rightarrow B \bar{B} \rightarrow\left(t W^{-}\right)\left(\bar{t} W^{+}\right) \\
& p p \rightarrow G \rightarrow t \bar{T}+b \bar{B}+c . c . \rightarrow t\left(\bar{t} Z+\bar{t} h+\bar{b} W^{-}\right)+b\left(\bar{t} W^{+}\right)+c . c . \\
& p p \rightarrow G \rightarrow(t \bar{t}+b \bar{b}+q \bar{q})
\end{aligned}
$$

As can be seen here, most of these processes lead to events with top quark pairs and $W, Z$ or Higgs bosons in the final state. This is interesting as in the searches for RS KK gluon 

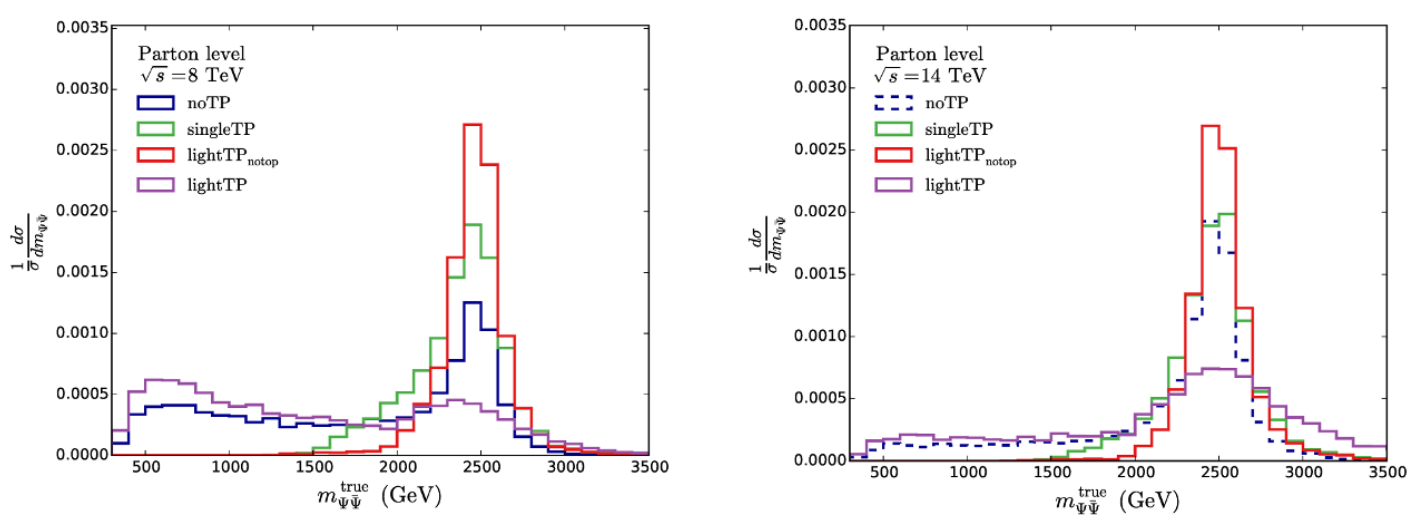

Figure 2. The true invariant mass distribution of the decay products of $G$ in $p p \rightarrow G \rightarrow$ $\psi \bar{\psi}, \Psi \bar{\psi}, \Psi \bar{\Psi}$ for $M_{G}=2.5 \mathrm{TeV}$ with $\sqrt{s}=8 \mathrm{TeV}$ (left) and $\sqrt{s}=14 \mathrm{TeV}$ (right).

resonances, the heavy gluon mass is kinematically reconstructed only from the tagged top quarks and the extra Higgs or vector bosons are not identified. Assuming that the top pairs can be reconstructed with good quality, one might expect the additional heavy bosons to have great qualitative impact on the reconstruction of the heavy gluon resonance at the LHC. Indeed that is case in some special kinematical regions, as we shall see.

We show in figure 3 the $m_{t \bar{t}}$ distribution at truth level at the $8 \mathrm{TeV}$ LHC for the extreme

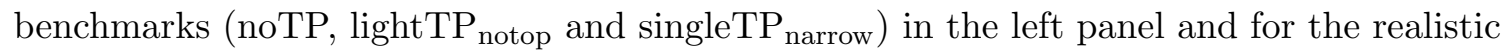
benchmarks (lightTP ${ }_{\text {narrow }}$ and lightTP) in the right one. The plots have been normalized to an integrated luminosity of $14.3 \mathrm{fb}^{-1}$. The corresponding results for the LHC14 and $300 \mathrm{fb}^{-1}$ of data are shown in figure 4. We see that the shape of simple Breit-Wigner type resonance is largely distorted especially for all models with new channels. These huge effects can be easily understood by the peculiar topology of top partner decays, which, as shown in eq. (3.1), leads to extra particles in the final state besides the top pairs. The invariant mass of most of the top-quark pairs produced from the $G$ decays is, therefore, much smaller than the $G$ mass as can be clearly seen in figures 3 and 4 . These results suggest that a purely $t \bar{t}$ resonance search would not be efficient at reconstructing the heavy gluon in the more general case of the $\mathrm{MCH}_{5}$ parameter space. We investigate this effect in the reconstructed sample in section 6 .

\section{Current bounds}

Both the ATLAS and CMS collaborations search for heavy resonances decaying in $t \bar{t}$ in both 7 and $8 \mathrm{TeV}$ data using the reconstructed top quark pair invariant mass $\left(m_{t \bar{t}}\right)$. These searches are sensitive to new resonances decaying to top quark pairs of various resonance widths, including narrow $Z^{\prime}$ bosons and broader heavy gluons. In the case of high-mass resonances, the use of jet substructure techniques to efficiently capture boosted top quarks or $\mathrm{W}$ bosons is crucial to achieve a good background rejection and efficient event reconstruction. All possible decay channels are considered in these studies: all-hadronic, lepton + jets and dilepton topologies. 

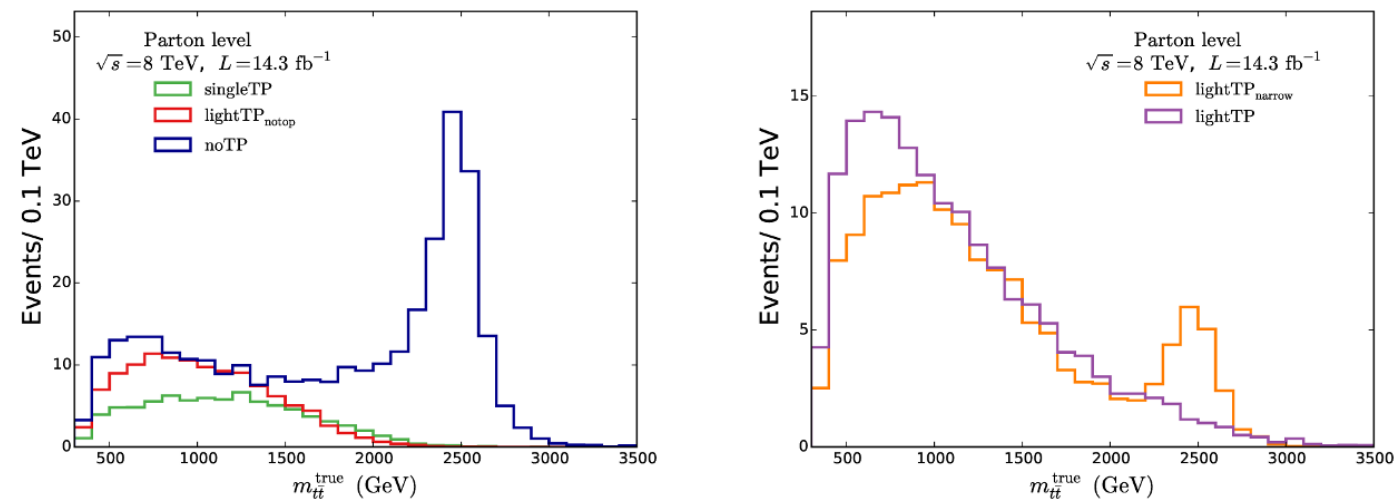

Figure 3. True (partonic) invariant mass for the $t \bar{t}$ pairs in $p \bar{p} \rightarrow G \rightarrow t \bar{t}+X$ for the noTP,

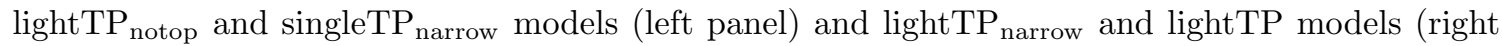
panel). The plots correspond to $M_{G}=2.5 \mathrm{TeV}$ at the $8 \mathrm{TeV} \mathrm{LHC}$.
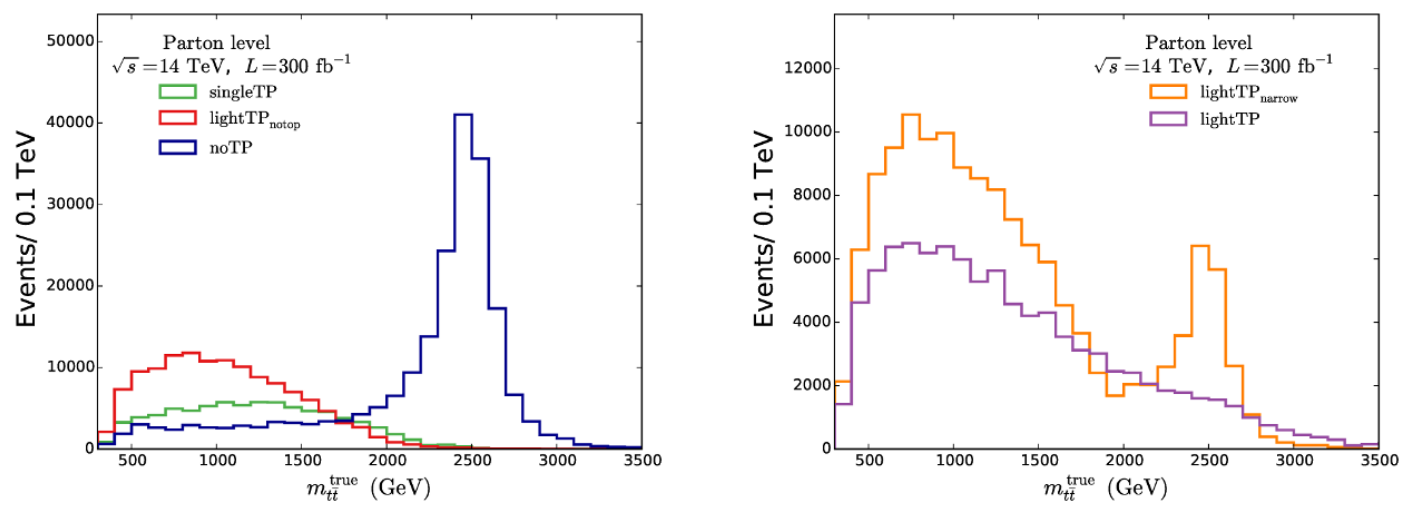

Figure 4. True (partonic) invariant mass for the $t \bar{t}$ pairs in $p \bar{p} \rightarrow G \rightarrow t \bar{t}+X$ for the noTP,

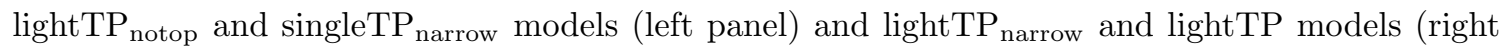
panel). The plots correspond to $M_{G}=2.5 \mathrm{TeV}$ at the $14 \mathrm{TeV}$ LHC.

Searches in the dilepton decay mode are performed by both ATLAS [38] and CMS [39]. In the former case the results are based on the LHC7 data, and the exclusion limits reach to roughly $1 \mathrm{TeV}$, depending on the signal model considered. In the latter case $8 \mathrm{TeV}$ data is used and the limit goes up to $1.8 \mathrm{TeV}$. Searches in the all-hadronic decay mode make use of top-tagging techniques. The latest results from CMS include $19.6 \mathrm{fb}^{-1}$ of LHC8 data and RS KK gluons are excluded up to $1.8 \mathrm{TeV}$ [40]. The latest results from ATLAS analyze $4.7 \mathrm{fb}^{-1}$ of LHC7 data, excluding KK gluon with masses below $1.62 \mathrm{TeV}$ [41].

Searches in the lepton + jets channel are also carried out by ATLAS [4] and CMS [5] with LHC8 data. ATLAS excludes KK gluons with masses up to $2.0 \mathrm{TeV}$ using $14.3 \mathrm{fb}^{-1}$ of integrated luminosity. CMS, using $19.6 \mathrm{fb}^{-1}$, excludes KK gluons up to $2.5 \mathrm{TeV}$, with a slight difference in the signal model with respect to ATLAS. To date these searches yield the strongest bounds on the RS KK gluon.

Since our focus is on disentangling the different effects of top partners on the heavy gluon searches, and not on improving the existing analysis, in this work we will consider 
only one of these searches, namely the lepton+jets analysis performed by the ATLAS collaboration. Because ATLAS and CMS employ somewhat different top reconstruction methods and have slightly different signal models, ${ }^{1}$ the results based on either of these methods would differ quantitatively, but qualitatively the shape of the distributions are expected to be similar.

We would like to make an important comment regarding the interplay between vector resonance and top partner searches. The standard production of top partners at the LHC is either pair production through QCD interactions or single production involving modeldependent couplings. Several experimental searches for top partners have been performed by ATLAS [3] and CMS [2, 42] with LHC7 and LHC8 data. The resulting lower bounds on the top partner masses depend on the channel and the assumptions on the branching ratios but they are typically in the $600-800 \mathrm{GeV}$ region. These analyses are quite inclusive in the final states but mostly consider QCD pair production as they are not yet sensitive to single production (see $[43,44]$ for a discussion of the relevance of single production in these searches). In the presence of a heavy gluon, there is an extra contribution to pair and single production of top partners mediated by the s-channel exchange of $G$. Thus, the current limits on top partners are expected to be more stringent than the ones reported above. For the benchmark models considered here, however, the event rate from a heavy gluon resonance contributes to the signal without running into serious conflict with existing experimental bounds. Choosing a mass of $1 \mathrm{TeV}$ for the lightest top partners yields an estimate of $20.7 \mathrm{fb}$ for the total cross section (conservatively including a K-factor of 1.3) while the current limit from CMS at this mass point is $23.7 \mathrm{fb}$.

\section{Method}

We will now describe the analysis procedure that we have followed in generating and analyzing the signal events for heavy gluon production at the LHC8 and LHC14. Throughout our analysis, we adhere to the search strategies developed by ATLAS [4], designed with the RS KK gluon model in mind, as closely as possible. The justification for the choices of specific analysis cuts as well as the use of the statistical tools for exclusion limits employed here are the same as those employed by ATLAS. In particular, we focus on the lepton+jets reconstruction mode.

\subsection{Event generation}

The implementation of the signal models was performed using Feynrules [45]. The production of samples was done with MadGraph/MadEvent [46] v.1.5.3 interfaced with Pythia [47] v. 6.426 for showering and hadronization. For the SM $t \bar{t}$ production, we use MLM matching [48] with up to one additional hard parton. We use the default tunes of

\footnotetext{
${ }^{1}$ The main source of the quantitative difference is that the ATLAS study only considers production of KK gluon resonance assuming a LO KK gluon production cross section, whereas CMS applies a flat K-factor of 1.3 to account for higher order effects. Meanwhile, the CMS observed limits on the KK gluon mass turned out to be higher than their expectation, whereas ATLAS observed limits were somewhat below their expectation.
} 
Pythia for the hadronization and underlying event model parameters, while for the matching scale in the $t \bar{t}$ sample we use $Q_{\text {cut }}=30 \mathrm{GeV}$ in both cases. ${ }^{2}$ Our MadGraph samples assume the CTEQ6L1 [49] PDF sets. While the signal cross sections are computed at LO, the background cross section is obtained with MadGraph normalized to the theoretical NNLO cross section of ref. [50]. We perform jet clustering using the FASTJET [51] implementation of the anti- $k_{T}$ algorithm [52].

The main background process is the irreducible SM $t \bar{t}$ after imposing all cuts described in the next section. The $W+$ jets and pure multi-jet QCD background events are difficult to simulate reliably, but we expect that they are efficiently suppressed by our cut procedure, in particular by the mini-Isolation cut and top tagging.

Not having at our disposal a reliable tool to estimate the response of the detector, a semi-realistic simulation of the hadronic final states would not be useful. Therefore we decided not to include the effects of pileup and underlying event in our analysis, as well as detector effects, and we stopped at the hadronization level. We treat electrons and muons together in order to get an estimate of the kinematical acceptance. Neither do we attempt to include uncertainties of the background. Consequently, this article does not present a fully realistic analysis but rather demonstrates the impact of new vector-like quarks on heavy gluon searches.

\subsection{Analysis details}

The analysis of the samples of simulated events is performed on the stable final-state particles using a custom analysis tool aimed at mimicking the ATLAS $\ell+$ jets search. The event selection is designed to tag $p p \rightarrow t \bar{t}$ events with subsequent decay $t \bar{t} \rightarrow b \bar{b} j j \ell \nu_{\ell}$. The expected kinematics of the top-quark decay products is characterized by two event topologies. In the first category, the $t \bar{t}$ pair is produced near the kinematic threshold, resulting in a topology where each parton is matched to a single jet (resolved topology). In the second category, each top quark is produced with a high Lorentz boost, resulting in collimated decay products that may be clustered into a single jet (boosted topology). The transition between the resolved and boosted topologies occurs around $m_{t \bar{t}}=1 \mathrm{TeV}$. The resolved and boosted selections are now discussed in detail.

The physics object selection criteria closely follow those used in the ATLAS $\ell+$ jets search, the main exceptions being the treatment of detector effects. Charged leptons are required to be mini-isolated [53],

$$
\operatorname{mini}-\mathrm{ISO} \equiv \frac{p_{T}^{\ell}}{p_{T}^{\text {cone }}}>0.9, \quad \Delta R(\ell, \text { track })<\frac{10 \mathrm{GeV}}{p_{T}^{\ell}},
$$

where mini-ISO is the lepton isolation observable of ref. [54] and $p_{T}^{\text {cone }}$ is scalar sum of all the charged tracks with $p_{T}>1 \mathrm{GeV}$, including the hard lepton, that fulfill the $\Delta R(\ell$, track $)$ requirement shown in eq. (5.1).

Small-radius jets are clustered from all final-state particles except neutrinos using the anti- $k_{T}$ algorithm with $R=0.4$. Only jets with $p_{T}>25 \mathrm{GeV}$ and $|\eta|<2.5$ are used.

\footnotetext{
${ }^{2}$ For the parton separation parameter of the MLM matching procedure in MadGraph, we use xqcut = $20 \mathrm{GeV}$.
} 
Large-radius jets are clustered in a similar way, but with a large radius $R=1.0$. These large-radius jets are required to have $p_{T}>350 \mathrm{GeV}$ and $|\eta|<2.5$.

We use a simple-minded algorithm for $b$-tagging in which an anti- $k_{T} R=0.4$ jet is matched to the corresponding $b$ parton. The typical performance of experimental $b$ tagging was simulated by applying a flat $b$-tagging efficiency of 0.7 . For the purpose of this analysis, we define a transverse missing energy vector, $E_{T}^{\text {miss }}$, to be the vector sum of all the neutrino transverse momenta in the event. The transverse mass is defined as $m_{T}=\sqrt{2 p_{T} E_{T}^{\text {miss }}(1-\cos \Delta \phi)}$, where $p_{T}$ is the transverse momentum of the charged lepton and $\Delta \phi$ is the angle in the transverse plane between the charged lepton $p_{T}$ and the missing transverse momentum.

Events are preselected by requiring exactly one mini-isolated electron or muon with $p_{T}>25 \mathrm{GeV}, E_{T}^{\text {miss }}>20 \mathrm{GeV}$ and $E_{T}^{\text {miss }}+m_{T}>60 \mathrm{GeV}$. Events are also required to pass either the boosted or resolved selections. In the Boosted Selection, events contain at least one anti- $k_{T} R=0.4$ jet and at least one anti- $k_{T} R=1.0$ jet. The highest $p_{T}$ small radius jet within a distance $\Delta R_{j \ell}<1.5$ from the lepton is deemed the $b$-jet of the leptonically decaying top. The fat jet must be well separated from the lepton and selected b-jet: $\Delta \phi_{J \ell}>2.3$ and $\Delta R_{J j}>1.5$. Two additional requirements on the substructure of the fat jet are made, the so-called ATLAS- $d_{12}$ tagger [53], which consists of the following cuts:

$$
\sqrt{d_{12}}>40.0 \mathrm{GeV}, \quad m_{j}>100 \mathrm{GeV} \text {. }
$$

The $m_{j}$ is the fat jet mass, ${ }^{3}$ and $\sqrt{d_{12}}=\min \left(p_{T, 1}, p_{T, 2}\right) \times \Delta R_{12}$ is the $k_{T}$ measure at the last step of large-radius jet clustering with a $k_{T}$ algorithm, where $p_{T, i}$ are the transverse momenta of the two subjets at the last step of fat jet clustering and $\Delta R_{12}$ is the plane distance between them. Boosted top quark decays are characterized by symmetric splittings $\sqrt{d_{12}} \approx m_{t} / 2$, whereas background QCD jets tend to have much smaller $d_{12}$.

In the Resolved Selection, events contain at least four small-radius jets with $|\eta|<2.5$ and $p_{T}>25 \mathrm{GeV}$, or only three small-radius jets if one of those jets has a mass greater than $60 \mathrm{GeV}$. The cuts and other kinematical constraints are summarized in table 2.

The $t \bar{t}$ invariant mass, $m_{t \bar{t}}$, is computed from the four-momenta of the two reconstructed top quarks. For the leptonically decaying top quark, the longitudinal momentum of the neutrino, $p_{z}$, is computed by imposing the $W$ boson mass constraint, $\left(M_{l \nu}=\right.$ $M_{W}=81 \mathrm{GeV}$ ), and solving the resulting quadratic equation. This information is sufficient to reconstruct the neutrino momentum, modulo a quadratic ambiguity. In the case that the solutions are complex, the magnitude of $E_{T}^{\text {miss }}$ is reduced to the point where $m_{T}\left(l, E_{T}^{m i s s}\right)=m_{W}$. In the case where we obtain two solutions, both solutions are tried.

For the resolved reconstruction, a $\chi^{2}$ algorithm is used to determine the correct assignment of jets to top quark candidates, using as constraints the top quark and $W$ boson masses and other kinematic properties of the signal process. All possible permutations for three or more small radius jets are tried and the permutation with the lowest $\chi^{2}$ is used to compute the $m_{t \bar{t}}$ distribution. This method is optimized for events containing tops and

\footnotetext{
${ }^{3}$ Strictly speaking, ATLAS uses $m_{j}^{\text {trim }}$, which is the trimmed fat jet mass with the trimming parameters $R_{\text {trim }}=0.3$ and $f=0.05$ (see ref. [55] for more details).
} 


\begin{tabular}{|c|c|}
\hline Selection & Cuts \\
\hline $\begin{array}{l}\text { Kinematic } \\
\text { and } \\
\text { acceptance }\end{array}$ & $\begin{array}{c}\text { lepton } p_{T}>25 \mathrm{GeV},|\eta|<2.5 \\
\geq 2 \text { jets } p_{T}>25 \mathrm{GeV},|\eta|<2.5 \\
\quad \text { tagged } b \text {-jets } \geq 1 \\
\text { missing energy }(\nu) E_{T}^{\text {miss }}>20 \mathrm{GeV} \\
\text { transverse mass } m_{T}+E_{\mathrm{T}}^{\text {miss }}>60 \mathrm{GeV} \\
\text { lepton isolation mini-ISO }>0.9\end{array}$ \\
\hline $\begin{array}{l}\text { Resolved selection } \\
\text { for } \# \text { jets }>2 \text {, } \\
1 b \text {-jet required }\end{array}$ & $\begin{array}{c}\geq 4 \text { jets } p_{T}>25 \mathrm{GeV},|\eta|<2.5 \text { or } \\
3 \text { jets } p_{T}>25 \mathrm{GeV},|\eta|<2.5 \& \\
\geq 1 \text { jets } m_{j}>60 \mathrm{GeV}\end{array}$ \\
\hline $\begin{array}{l}\text { Boosted selection } \\
1 b \text {-jet }+1 R=1.0 \text {-jet }\end{array}$ & $\begin{array}{c}m_{\text {jet }}>100 \mathrm{GeV} \& \sqrt{d_{12}}>40 \mathrm{GeV} \\
p_{T}^{\text {jet }}>350 \mathrm{GeV} \& \quad\left|\eta_{\text {jet }}\right|<2.5 \\
\Delta \phi(\text { jet }, l)>2.3 \& \quad \Delta R(\text { jet }, b)>1.5\end{array}$ \\
\hline
\end{tabular}

Table 2. Selection cuts in the semileptonic $t \bar{t}$ channel.

$W$ 's which are not too energetic, as in the case of the $t \bar{t}$ events with top quark invariant masses smaller than about $1 \mathrm{TeV}$. In this case, leptons will be isolated, and there is good match between jets and parton momenta.

For the boosted reconstruction, there is no ambiguity in the assignment of jets. The hadronically-decaying top quark is taken from the fat jet, while the leptonically decaying top quark momentum is formed from the neutrino solution, the lepton and the selected small-radius jet. The $m_{t \bar{t}}$ distribution is used for signal discrimination, after combining the resolved and boosted analyses.

\subsection{Statistical procedure}

To determine the expected reach for the five benchmark models in section 2, we use a binned Bayesian approach with a flat, positive prior on the signal cross section. We assume that the probability of measuring $n$ events in statistically uncorrelated bins is given by a Poisson distribution

$$
P(\{n\} \mid S, B)=\sum_{\text {bins }}\left[\frac{\left(S_{i}+B_{i}\right)^{n_{i}}}{n_{i} !} e^{-\left(S_{i}+B_{i}\right)}\right],
$$

where $B_{i}$ and $S_{i} \equiv \sigma_{\mathrm{sig}} \epsilon_{i} \mathcal{L}$ are the number of expected background and signal events in each bin. Here we regard $\sigma_{\text {sig }}$ as a free parameter in order to consider different signal production rates and fix $B$ and $\epsilon_{i}$ according to our expectations based on the Monte Carlo distributions. An upper limit for $\sigma_{\text {sig }}$ at confidence level $\mathrm{CL}=1-\alpha$ can be constructed by integrating the posterior probability,

$$
\mathrm{CL}=1-\alpha=\frac{\int_{0}^{\sigma^{\mathrm{CL}}} P\left(n \mid \sigma_{\mathrm{sig}} \epsilon_{\mathrm{sig}} \mathcal{L}, B\right) d \sigma_{\mathrm{sig}}}{\int_{0}^{\infty} P\left(n \mid \sigma_{\mathrm{sig}} \epsilon_{\mathrm{sig}} \mathcal{L}, B\right) d \sigma_{\mathrm{sig}}} .
$$




\begin{tabular}{|ccc|}
\hline$g_{K K}$ mass $[\mathrm{TeV}]$ & Resolved selection & Boosted selection \\
\hline 500 & $0.027(0.0351 \pm 0.0029)$ & $0.0004(0.00042 \pm 0.0001)$ \\
600 & $0.033(0.0400 \pm 0.0032)$ & $0.0009(0.00122 \pm 0.0003)$ \\
700 & $0.037(0.0440 \pm 0.0032)$ & $0.004(0.0039 \pm 0.0011)$ \\
900 & $0.036(0.0400 \pm 0.0032)$ & $0.019(0.0170 \pm 0.0022)$ \\
1000 & $0.035(0.0370 \pm 0.0028)$ & $0.026(0.0242 \pm 0.0022)$ \\
1300 & $0.031(0.0344 \pm 0.0024)$ & $0.039(0.035 \pm 0.0021)$ \\
1600 & $0.032(0.0304 \pm 0.0018)$ & $0.045(0.039 \pm 0.004)$ \\
1800 & $0.030(0.0289 \pm 0.0017)$ & $0.046(0.042 \pm 0.005)$ \\
2000 & $0.030(0.0286 \pm 0.0017)$ & $0.050(0.041 \pm 0.007)$ \\
2500 & $0.029(0.0293 \pm 0.0017)$ & $0.045(0.038 \pm 0.008)$ \\
\hline
\end{tabular}

Table 3. Acceptance $\times$ efficiency for $G \rightarrow t \bar{t}$ samples in the $\mu+$ jets channel. The ATLAS result is in parenthesis.

To obtain the expected limit on the signal cross section, we solve eq. (5.4) for $\sigma^{\mathrm{CL}}$ assuming $n=B$ and $\alpha=0.05$ (95\% exclusion).

\subsection{Comparison with ATLAS benchmark analysis}

ATLAS has searched for RS KK gluons using $14.3 \mathrm{fb}^{-1}$ of LHC8 data. As a sanity check, it is imperative for us to compare the results of our analysis with these published results for the same benchmark model before proceeding to apply our analysis to the $\mathrm{MCH} 4_{5}$ benchmark set. The comparisons shown in figure 5 and table 3 indicate that we are indeed able to reproduce to a reasonable degree the results obtained by ATLAS for this benchmark model for the $\ell+$ jets analysis.

Here it is important to remind the reader that our event generation and analysis differs slightly from the procedure used by ATLAS. In particular, our simulation of SM $t \bar{t}$ background includes only the real emissions through matching with no contributions from the virtual part of the NLO diagrams. These higher order effects could have a great impact on the shape of the distributions, especially around the region of high $t \bar{t}$ invariant masses. As noted before, we do not apply a full detector simulation. Furthermore, in our statistical procedure we have neglected the effect of systematic uncertainties in the background. Therefore, some degree of discrepancy can be expected. Notice, however, the small differences will not affect the conclusion of this work, namely that semi-leptonic $t \bar{t}$ searches rapidly loose sensitivity once the decay into top partners are open. This is a generic statement in the sense that once the signal becomes elusive the signal-to-background ratio reduces by about two orders of magnitude, and this ratio is not expected to change significantly after detector smearing or inclusion of systematic effects.

\section{Results}

Now that we have introduced the main points of our analysis, we turn to a quantitative discussion of the results for our $\mathrm{MCH}_{5}$ set-up. The extended scenario under consideration 


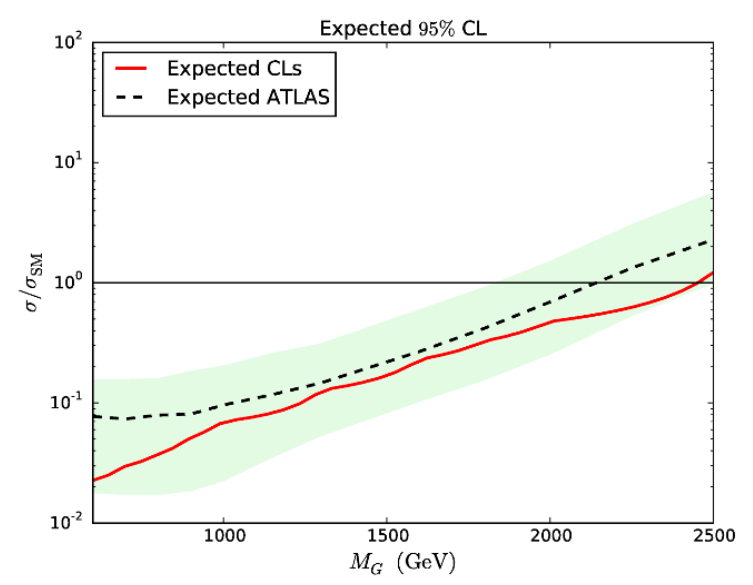

Figure 5. The 95\% confidence level limit on the heavy gluon production cross section divided by the expected $p p \rightarrow g_{K K} \rightarrow t \bar{t}$ production in the RS KK gluon model.

has fermionic partners of the top together with the heavy gluon that can be characterized by the two masses $M_{G}$ and $M_{\Psi}$ and the degree of compositeness $g_{c}$.

In this section, we probe our set of $\mathrm{MCH}_{5}$ benchmark models with the suite of $t \bar{t}$ resonance searches performed at the LHC8, as well as planned searches at the LHC14. As described in section 3, there are three challenges in obtaining a signal at the LHC for heavy gluons in the $\mathrm{MCH} 45$ scenario:

- Because the top partners are already constrained to lie above $800 \mathrm{GeV}$, heavy gluon production is suppressed due to smaller available phase space.

- The signal final-state is characterized by a top-quark pair and up to two extra massive gauge or Higgs bosons. This difference with respect to the pure $t \bar{t}$ final state systematically shifts events to values of $m_{t \bar{t}}^{t r u e}$ much smaller than the $G$ mass.

- The large multiplicity of available channels implies that in most of the cases the heavy gluon is a rather broad resonance, which leads to a strong departure from the narrow width approximation.

It is clear from the discussion above that the $t \bar{t}$ resonance searches are likely to have much reduced sensitivity to the heavy gluon in the $\mathrm{MCH}_{5}$ model. However, a quantitative statement requires a more careful treatment as reconstructing the heavy gluon mass involves measuring top quarks in a wide range of transverse momentum with techniques that were optimized for the $t \bar{t}$ final-state hypothesis. In this situation, there are two issues which might arise:

- The sensitivity to any resonance search depends on the number of events available for analysis, which is affected by the overall efficiency of each selection. The precise interplay of the resolved and boosted analyses is likely to be highly process dependent and requires a dedicated study. 
- The additional particles in the final state beyond $t \bar{t}$ can have a significant impact on the ability to experimentally resolve the underlying parton-level distributions of the top kinematic observables. The issue of resolution is inseparable from the genuine new physics effects that distort the invariant mass distributions of top pairs, as a wrongly reconstructed top will lead to an incorrect estimate of the kinematic properties of the truth-level objects.

With this in mind we are going to study whether and to what extent the new decay topologies might imply any observable excess in physical distributions. For the purpose of heavy gluon reconstruction, we find the analysis performed by the ATLAS collaboration for their KK gluon benchmark points at the LHC8 run to be adequate to illustrate the general situation without much loss of generality.

While our focus in this section is for most of the part on physical distributions, we find it instructive to look back at the parton-level truth information for events that pass our full set of reconstructions, as this gives a feeling on how much mass degradation is due to misreconstruction effects versus genuine new physics effects. In order to disentangle these two effects, we consider the $t \bar{t}$ invariant mass resolution defined as

$$
\epsilon \equiv\left(m_{t \bar{t}}^{\mathrm{rec}}-m_{t \bar{t}}^{\mathrm{truth}}\right) / m_{t \bar{t}}^{\text {truth }}
$$

with $m_{t \bar{t}}^{\text {reco }}\left(m_{t \bar{t}}^{\text {truth }}\right)$ being the invariant mass of the reconstructed (truth) top-quark pair. Here, $\epsilon$ is computed on an event-by-event basis for events that pass the selection cuts. The resolution should vanish for a perfectly reconstructed top-quark pair.

In addition to the LHC8 searches, future data taking and enhanced analyses at $14 \mathrm{TeV}$ will greatly extend the expected coverage of searches for heavy-colored particles decaying to $t \bar{t}$. Here, we consider the impact of two different analyses. The first one is similar to the lepton+jets search presented in ref. [56] by the ATLAS collaboration. We have performed our own version of this analysis in a manner identical to that employed above for the LHC8 by following ATLAS as closely as possible. A full signal+background study using partonlevel truth information is also provided as a limiting example of the expected reach at the $14 \mathrm{TeV}$ LHC, independently of any particular experiment or top reconstruction method.

The second analysis attempts to reconstruct the $G$ mass by making use of all jets in the final state. Among the main issues one might face for such an analysis is the challenge of identifying and tagging many jets in the very intense hadronic activity that this kind of events produce. As we focus here on the present experimental status of the heavy gluon searches, we do not consider the reconstruction of the top partners in full and leave a detailed analysis of these signatures for future work. Rather, we content ourselves with pointing out a simple-minded analysis to extend the reach of these searches, in the case in which the heavy gluon is a relatively narrow resonance.

\subsection{Physical distributions}

To get an idea of how the new topologies affect the event selection, we study the performance of the selection cuts used by ATLAS. Figure 6 shows the efficiency for the Boosted and the final (Boosted+Resolved) selections on various models as a function of the heavy 

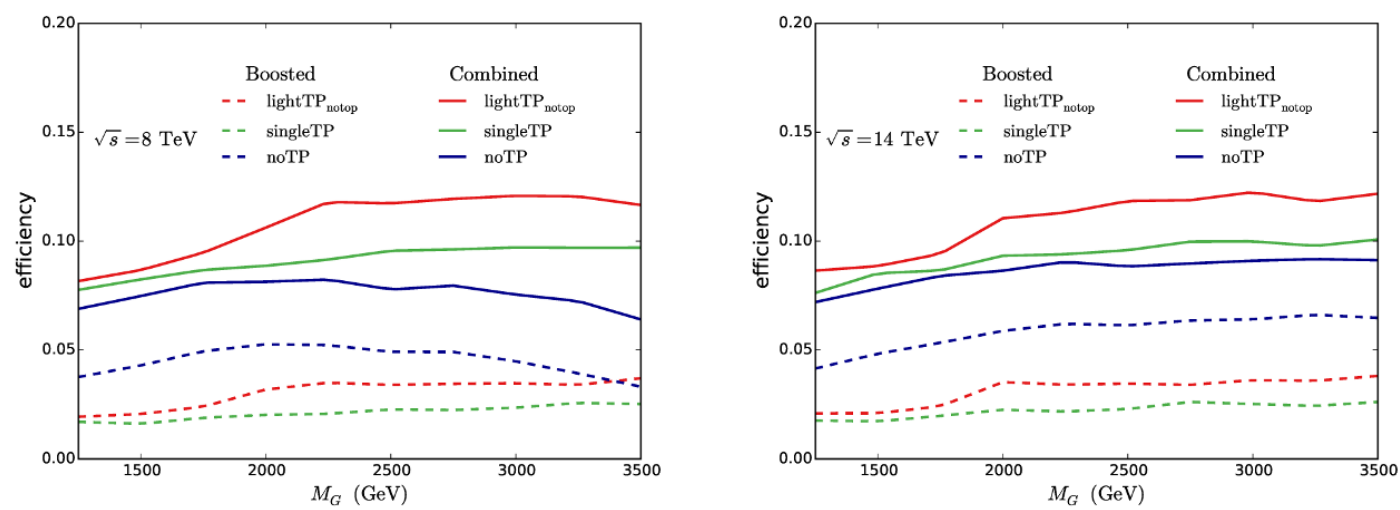

Figure 6. The selection efficiency as a function of $M_{G}$. Dashed lines show the boosted selection and solid lines the total selection efficiency. The plots correspond to $M_{\Psi}=1 \mathrm{TeV}$.

gluon invariant mass. Here we define the efficiency for the TP models as the passing fraction of reconstructed $t \bar{t}$ events in the lepton+jets final state. As expected, the Boosted selection is less efficient in the case with new channels than in the noTP model, because tops from top partner decays tend to have lower transverse momentum than those coming directly from heavy gluon decays, and the Boosted selection is optimized to tag tops at very high $p_{T}$. For the combined selection, however, the TP topologies can have higher pass rates than the noTP model. This is because the Resolved reconstruction is designed to reconstruct events with low $m_{t \bar{t}}$ and, hence, allows for more efficient reconstruction of the TP events. ${ }^{4}$ This result suggests that a purely boosted search for $t \bar{t}$ final states might not be the optimal strategy for models with top partners. Resolved analysis might still provide relevant information even as we increase the reach in mass.

In figure 7 (left), we show the distributions of reconstructed $t \bar{t}$ invariant mass, $m_{t \bar{t}}^{\text {rec }}$, for the signal only and $M_{G}=2.5 \mathrm{TeV}$. The results are normalized to the LO production cross section obtained from MadGraph and $14.3 \mathrm{fb}^{-1}$ of integrated luminosity. While the noTP distribution shows the resonance structure corresponding to a heavy gluon with $M_{G}=2.5 \mathrm{TeV}$, the invariant mass distributions for the models with new channels are significantly distorted. Interestingly enough, although the reconstructed distributions are consistent with the parton-level distributions in section 3, we see that the number of events at high $t \bar{t}$ invariant mass is increased in the TP distributions compared to the partonlevel results. Indeed, the $t \bar{t}$ analysis appears to preferentially select $t \bar{t}$ solutions which reconstruct to form a larger invariant mass, which manifests itself in events with higher $m_{t \bar{t}}$ in comparison with figure 3. In the resolved analysis, this is because the extra particles in the final state can lead to a wrong assumption on the reconstructed top when determining the $b-W$ combination from genuine tops. In the boosted analysis, one fat jet can overcollect jets from the extra bosons which are not far away from the tops. Due to loose mass constraints employed in the top quark tagging algorithm, misidentification of this fat jet as

\footnotetext{
${ }^{4}$ However, the Resolved selection does not include a minimum quality cut on the $\chi^{2}$ distribution and as such does not guarantee that the invariant mass of the two reconstructed top quarks will reflect the invariant mass of the underlying truth-level tops with enough precision.
} 

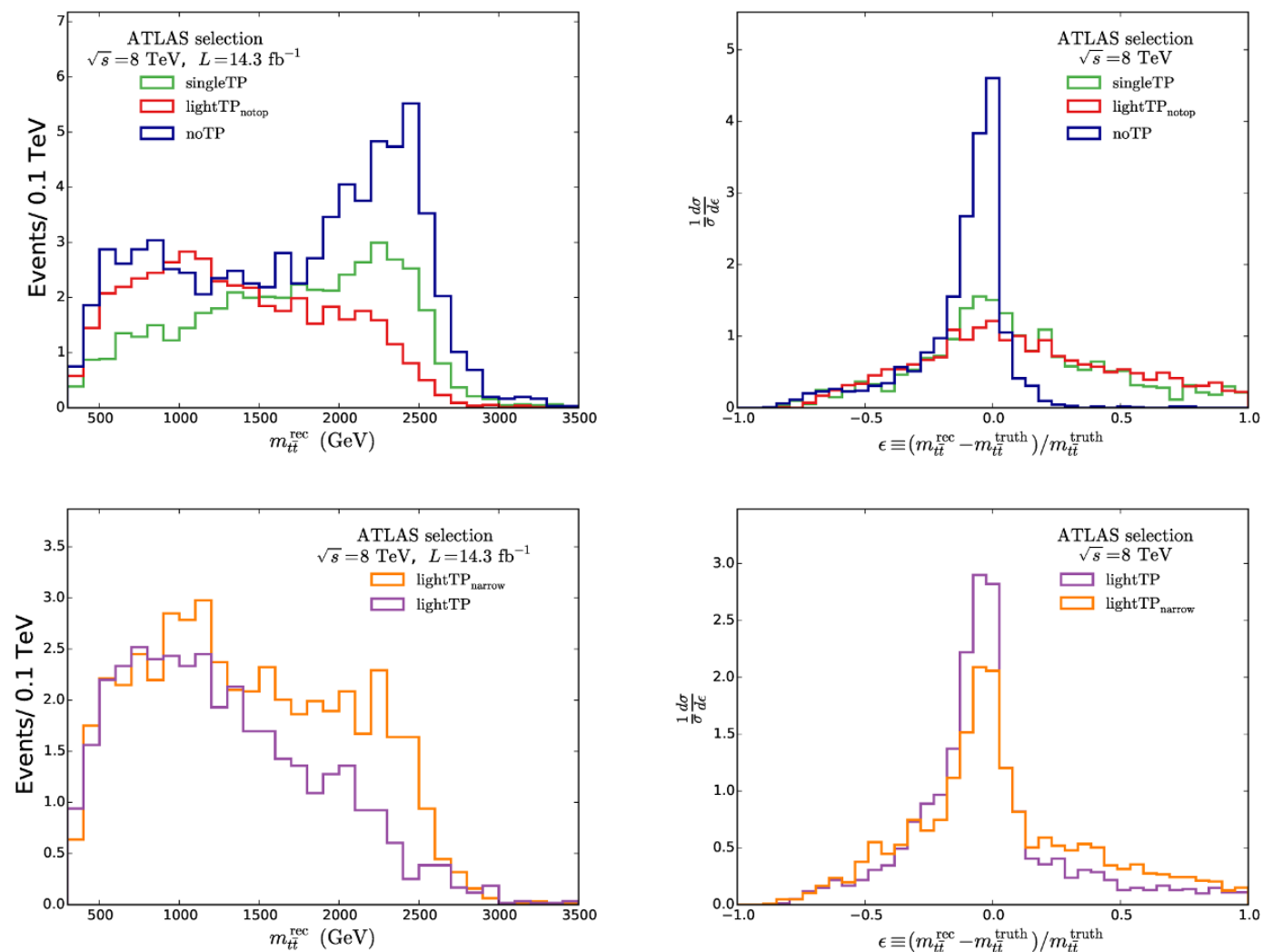

Figure 7. Left panels: the reconstructed $t \bar{t}$ invariant mass distribution in signal events with 14.3 $\mathrm{fb}^{-1}$ at $\sqrt{s}=8 \mathrm{TeV}$. Right panels: di-top invariant mass resolution. $m_{t \bar{t}}^{\text {truth }}$ denotes the true (partonic) invariant mass of the top-quark pairs, while $m_{t \bar{t}}^{\text {rec }}$ is the reconstructed invariant mass of the top-quark pairs using the ATLAS analysis. The plots correspond to $M_{G}=2.5$ and $M_{\Psi}=1 \mathrm{TeV}$ for the five benchmark models.

$t$ leads to an incorrect estimate of the kinematics of the top pairs. These fake candidates are legitimate as far as the semileptonic top-pair selection is concerned and in fact, even though they do not faithfully reflect the underlying truth-level tops from the hard process that originated them, they allow us to partially recover some of the signal. The use of more efficient top taggers would however go in the opposite direction, reconstructing distributions that are more similar to the partonic ones and therefore worsening the reach in the heavy gluon searches. To make the comparison with our parton-level results more evident, in figure 7 (right), we show distributions of the resolution $\epsilon$ for the same benchmarks. We can see that the resolution for the TP models (those with light top partners) is significantly worse than that for the noTP model.

Note that, although the used top taggers allow us to retain some of the signals at invariant masses near the heavy gluon mass, the distributions are still quite broad towards smaller values of the invariant mass. Since the SM top pair production rate falls steeply as a function of the invariant mass, the net effect of the new TP topologies is to shift the contributions to a mass range where one would expect a higher SM $t \bar{t}$ background. It is therefore worth investigating whether the new processes could nevertheless lead to 
a potentially interesting signal. In the left panels of figure 8 , we plot the total (signal + background) $m_{t \bar{t}}^{\text {rec }}$ distributions for the five different models and $M_{G}=2.5 \mathrm{TeV}$. In the right panels of figure 8, we focus on the area near the peak. As expected, we see that the generic form of the resonance in the noTP model is clearly visible with the peak located at about $M_{G}$. However, for the singleTP $\mathrm{P}_{\text {narrow }}$ benchmark point the bump is much less

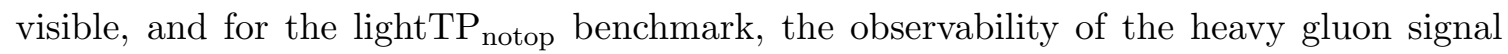
is severely diminished. A similar behavior is obtained for the lightTP and lightTP $\mathrm{Parrow}_{\mathrm{w}}$ models. Even though we only show results for a single mass point, we found that these features of the $t \bar{t}$ spectrum are generic for all mass points for which the decays into top partners are kinematically open. A priori, since the lepton+jets searches at $8 \mathrm{TeV}$ are less effective at reconstructing the heavy gluon in the $\mathrm{MCH} 4_{5}$ scenario compared with the RSlike KK gluon, we would expect the same features to also be present for a single $14 \mathrm{TeV}$ analysis. Indeed we have checked that a similar behaviour is obtained at the LHC14. We do not reproduce the precise plots here as they are qualitatively equal to the ones at $8 \mathrm{TeV}$. The result is that essentially all models with top partners result in a much distorted $t \bar{t}$ spectrum with a peak shifted to lower values of reconstructed $m_{t \bar{t}}$. As a consequence, the signal in these models is much less visible over the continuum SM $t \bar{t}$ than in the noTP case.

\subsection{Expected limits on heavy gluon mass}

The above results suggest that the $t \bar{t}$ resonance searches should indeed have reduced sensitivity to exclude certain regions of the $\mathrm{MCH}_{5}$ parameter space. We use a Bayesian statistical method to extract the $95 \%$ C.L. upper limits on the $p p \rightarrow G$ cross section as described in section 5.3. For each $G$ mass point and benchmark model, we generate $10^{4}$ signal events using our MadGraph+Pythia chain and scale them to an integrated luminosity of $14.3 \mathrm{fb}^{-1}$ for $\sqrt{s}=8 \mathrm{TeV}$ and $300 \mathrm{fb}^{-1}$ for $\sqrt{s}=14 \mathrm{TeV}$. For each of the mass points investigated, we apply our reconstruction in order to obtain a value for $\sigma \times B R \times \epsilon$ to be used in the Bayesian analysis. The results are shown by solid lines in figure 9 . The heavy gluon production cross sections are indicated by the dashed lines in figure 9 .

The performance of the $t \bar{t}$ search depends strongly on the final state topology. For $M_{G} \lesssim 2 \mathrm{TeV}$, decays into top partners are closed, and we return to the noTP case. For $M_{G} \gtrsim 2 \mathrm{TeV}$, the bounds are weakened considerably because 1) the decay channels into top partners open up, and this tends to dilute the signal over the SM $t \bar{t}$ background, and 2) the production cross section is reduced due to suppressed phase-space and departure from the narrow-width approximation. With $14.3 \mathrm{fb}^{-1}$ of $8 \mathrm{TeV}$ data, the bounds for realistic heavy gluons can be up to $\sim 400 \mathrm{GeV}$ less stringent than the bound for the heavy gluon in the noTP model. The increase in the center of mass energy to $14 \mathrm{TeV}$ and integrated luminosity to $300 \mathrm{fb}^{-1}$ makes a significant impact on the overall $\mathrm{MCH} 4_{5}$ model coverage. However, the differences between the models are still significant: realistic heavy gluons can be up to $1.5 \mathrm{TeV}$ lighter than the heavy gluon in the scenario considered by the experimental searches.

As we have already mentioned, future analyses -specifically those making use of a dedicated top tagging tool- might make things worse. An increase in the top reconstruction efficiency would make the distributions more similar to those found using truth-level tops, 

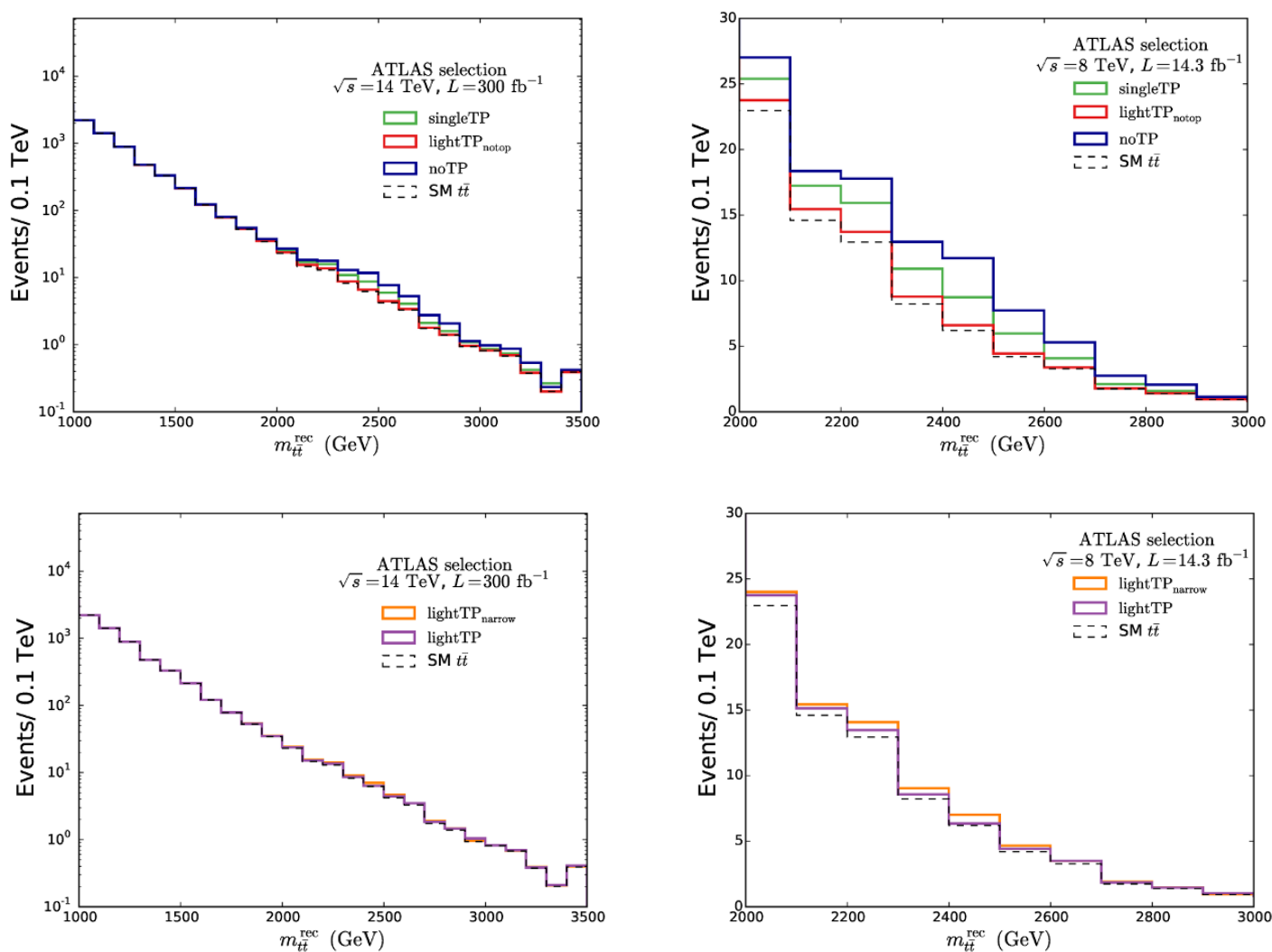

Figure 8. Reconstructed $t \bar{t}$ invariant mass distributions for heavy gluon production at the LHC with $\sqrt{s}=8 \mathrm{TeV}$. The solid histogram presents signal+background distribution, while the dashed histogram presents the $t \bar{t} \mathrm{SM}$ background. The right panels show the reconstructed invariant $t \bar{t}$ distribution focusing on the area near the would-be peak. The plots correspond to $M_{G}=2.5$ and $M_{\Psi}=1 \mathrm{TeV}$ for the five benchmark models. Events are reconstructed using the ATLAS analysis by requiring the combined selection.

where the differences between the models were more significant. A parton level study, where tops are reconstructed perfectly, can be used to set worst-case scenario limits. In figure 10 we show the expected bounds on the cross section $\sigma$ as a function of $M_{G}$ at $14 \mathrm{TeV}$ using only parton-level truth information. This example shows that the limit could be weakened by up to $2 \mathrm{TeV}$ with $300 \mathrm{fb}^{-1}$ at the LHC14.

This makes clear that the LHC discovery potential on heavy gluon resonances may be crippled by a limited choice of search regions that may render impractical those analyses based on the $t \bar{t}$ hypothesis. Nevertheless, several properties of the top partners set them apart from ordinary top jets, and dedicated searches could very likely extract the signal from the SM background as in refs. [29-33]. Below we point out some features of the signal that could be targeted by experiments in order to increase the sensitivity. The discussion is concise and qualitative; a more quantitative study is beyond the scope of this paper and will be attempted elsewhere. 

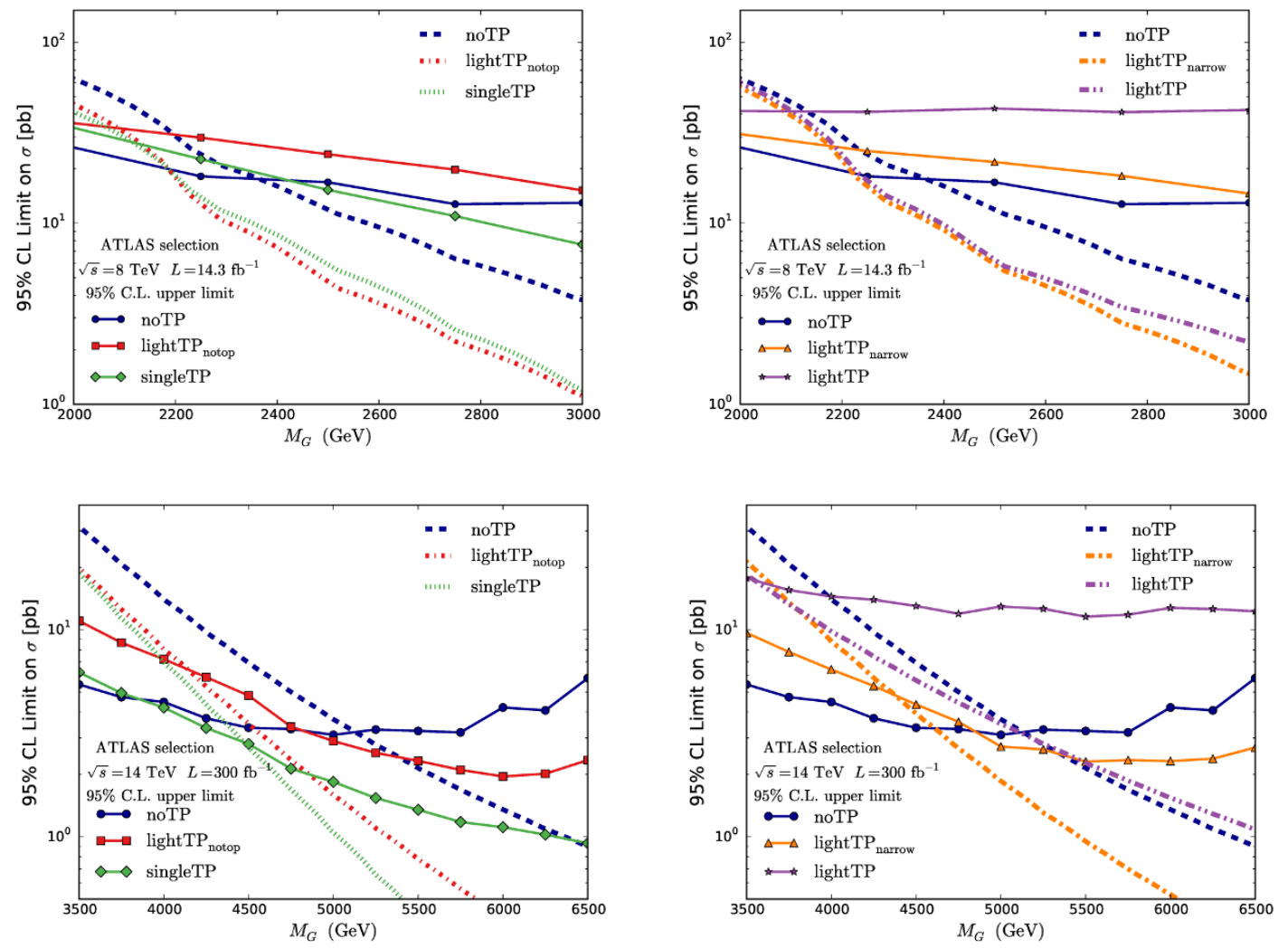

Figure 9. The 95\% confidence level limit (solid lines) on the heavy gluon production cross section as a function of $M_{G}$ is given for the five benchmark models. The theoretical cross sections are shown by the dashed lines.
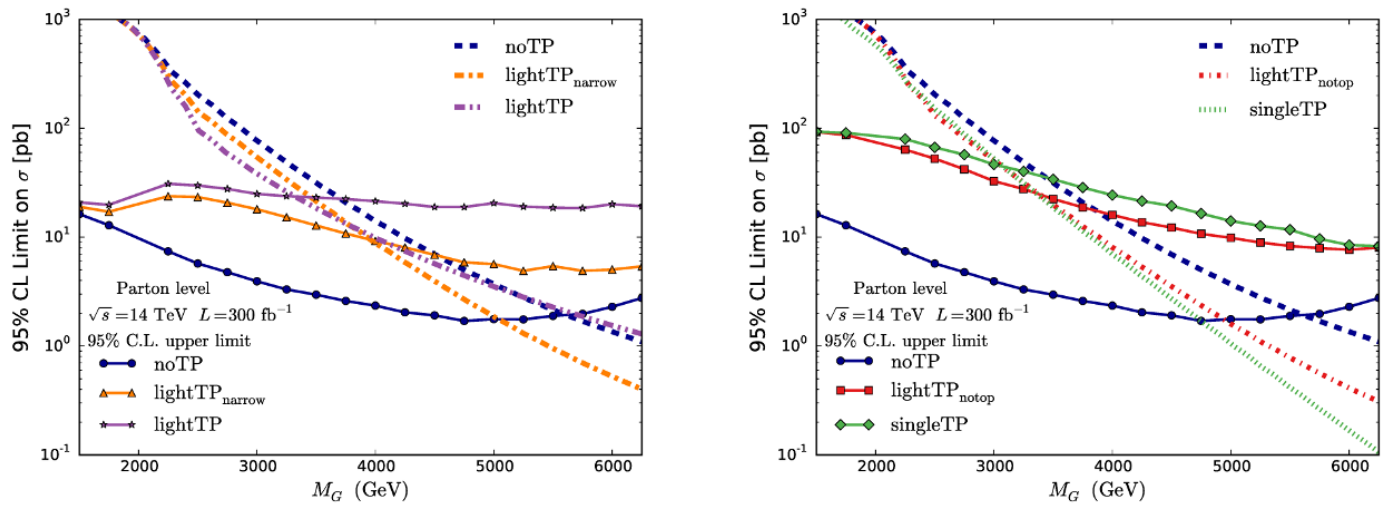

Figure 10. The $95 \%$ confidence level limit (solid lines) on the heavy gluon production cross section as a function of $M_{G}$ is given for the five benchmark models using the parton-level information. The theoretical cross sections are shown by the dashed lines.

\subsection{Recovering the signal}

With such low signal sensitivity it is interesting to attempt a more tailored search that would have an increased sensitivity. We try to be as inclusive as possible and determine 
whether a search that was not optimized to look for secondary resonances (the top partners) could still discover the heavy gluon. For the the mass of the top partners considered in this paper, the extra bosons are typically boosted and their decay products are very collimated and can be caught within a fat jet of large radius. Therefore, we slightly change the analysis by including the jets resulting from the hadronic decays of the extra bosons in the top partner cascade decay. Using the same selection cuts as above but without discarding the sub-leading fat jets with $p_{T}>200 \mathrm{GeV}$ we simply construct the invariant mass of the $t \bar{t}$ system and any sub-leading fat jet found by the following procedure. The ATLAS $t \bar{t}$ reconstruction is first used to select the event and reduce every final state to the ditop topology. Next, we find all anti- $k_{T} R=1.0$ jets, $j_{i}$, which are well separated from all the jets that are part of the reconstructed $t \bar{t}$ system,

$$
\begin{aligned}
& \text { Boosted selection : } \Delta R\left(J, j_{i}\right)>1.0, \Delta R\left(j_{\text {sel }}, j_{i}\right)>1.4 \\
& \text { Resolved selection : } \Delta R\left(j, j_{i}\right)>1.4,
\end{aligned}
$$

where $j_{\text {sel }}(J)$ are the selected small-radius (large-radius) jet in the boosted reconstruction sample, and $j$ is any of the selected small-radius jets in the resolved reconstruction sample. We then combine the four momenta of these jets with the four momenta of the reconstructed leptonically and hadronically decaying top quarks. The candidate invariant mass, $M_{G}^{\text {reco }}$, is computed from the four momenta of all physics objects in the event,

$$
m_{G}^{\mathrm{reco}}=\left(p_{t_{1}}+p_{t_{2}}+\sum_{i \notin t_{1}, t_{2}} p_{i}\right)^{2}
$$

where $p_{t_{i}}, i=1,2$ are the four-momenta of the top candidates, $p_{i}$ are the four-momenta of the $j_{i}$ and the sum runs over the $j_{i}$ which satisfy eq. (6.2).

Figure 11 shows the distributions of the reconstructed invariant $G$-mass using both methods; we show the distributions for the noTP model (red) and the lightTP narrow $_{\text {model }}$ (blue) and $M_{G}=2.5 \mathrm{TeV}$. Also for comparison we show in dashed lines the distributions using the ATLAS analysis. These histograms show that, as expected, the noTP model is not sensitive to the different reconstruction methods but the lightTP ones can greatly benefit from the modified analysis. Indeed, this new analysis provides a more efficient method to reconstruct the invariant mass of the heavy gluon by recovering some of the heavy vector and Higgs bosons coming from the top partners. Furthermore, we can use this behaviour in the invariant mass to search for narrow resonances in the background from continuum top partner pair production.

In figure 12 we present the results for $\sqrt{s}=14 \mathrm{TeV}$ and a total integrated luminosity of $300 \mathrm{fb}^{-1}$. We show the $95 \%$ C.L. upper limit on the heavy gluon production cross section as a function of $M_{G}$ according to the ATLAS lepton+jets analysis for the noTP sample (blue) and for the lightTP $\mathrm{T}_{\text {notop }}$ sample (red). We also show in the same figure for comparison the bound using the modified lepton+jets analysis for the same samples. As expected, no significant difference is seen on the noTP sample when using the modified analysis. The results presented in figure 12 allow us to conclude that a sizeable improvement in the 

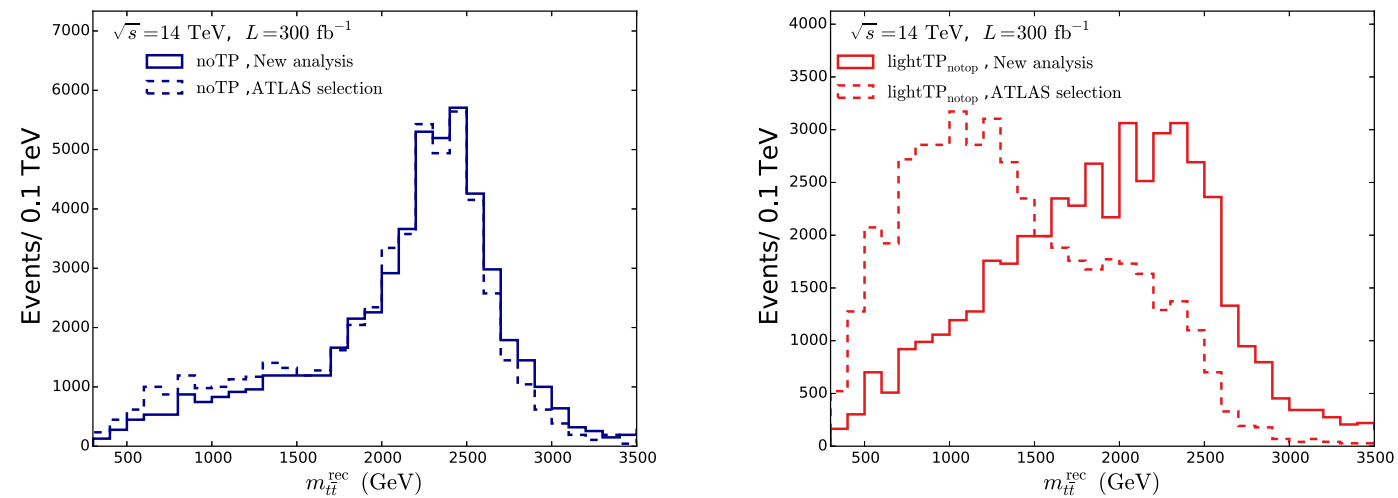

Figure 11. Reconstructed $G$ invariant mass distributions for noTP model (left) and lightTP ${ }_{\text {notop }}$ model (right). The plots correspond to $M_{G}=2.5 \mathrm{TeV}$ and $M_{\Psi}=1 \mathrm{TeV}$ in the $\mathrm{MCH}_{5}$ model with gc4 couplings.

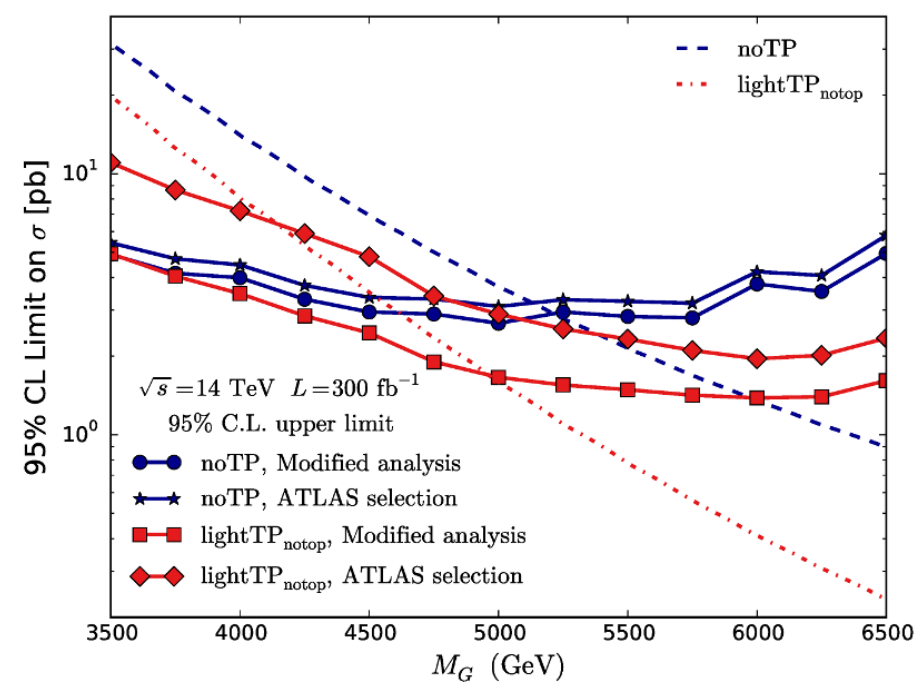

Figure 12. The $95 \%$ confidence level limit (solid lines) on the heavy gluon production cross section as a function of $M_{G}$ according to the ATLAS lepton+jets analysis and our modified analysis. The theoretical cross sections are shown by the dashed lines.

bound can be achieved by including the extra hadronic activity in $t \bar{t}$ resonance searches. Furthermore it is clear that further improvement can be attained by performing full-fledged di-top-partner resonance searches, especially for the case of narrow resonances.

\section{Conclusions and outlook}

Natural composite Higgs models generically contain both fermionic and vector resonances. The former, called top partners, are expected to couple strongly to the electroweak boson and gluon partners, and this expectation is quantitatively confirmed by arguments based on holography. The combination of bounds from direct and indirect searches and naturalness 
arguments lead to the plausible and more viable scenario in which there is a small hierarchy between the masses of the spin-half and spin-one resonances. This hierarchy suggests an important change in the way we look for the vector resonances at the LHC experiments. In this work we have focused on searches for a heavy gluon but similar arguments apply to electroweak vector resonances. The present experimental analyses are not geared for the case in which the heavy gluon decays with a large decay ratio into top partners, as we have explicitly demonstrated in this paper. The final effect on the heavy gluon exclusion limits is model dependent but we find a qualitative decrease in the experimental bounds on the heavy gluon mass in the $\mathrm{MCH} 4_{5}$ model. This means that even the simplest composite Higgs scenarios provide highly non-standard gluon partner signals that elude existing search strategies aimed at the RS-like KK gluon, composite Higgs models or their close variants.

Finally, a few words on the interplay of our results with top partner direct searches is in order. While near future single production searches can potentially rather quickly reach the $2 \mathrm{TeV}$ scale (see e.g. [57] using state of the art substructure methods [58, 59]) they are however rather model dependent. On the other hand the more robust searches via pair production are very limited in their reach (see e.g. recent discussion of reach [60]). Indeed, the models that we have considered in this work are not excluded so far by the direct searches. Consequently, one may ask whether one can use the new resonance-toppartners production as a discovery channel. The answer should be in principle positive (see [26] for a detailed discussion of this point in the context of holographic composite Higgs models). One can envision two ways to go about it, the first was already described by us in the previous section, namely, increasing the sensitivity via cutting hard on the activity in the event, say by looking at extra hadronic activity. Similarly one can look at extra contributions to the transverse mass or energy from leptonic and missing energy type of deposition. As this should be rather efficient way to increase the signal-to-background ratio one might be able to extend the pair production reach and to allow for an early discovery. Finally, if the vector resonance is narrow (as could happen in composite models for the coloured resonances and especially for the electroweak ones), then one may hope to be able to significantly extend the reach to regions which are well beyond that of the direct production regime.

\section{Acknowledgements:}

JJ would like to thank the Weizmann theory group for their hospitality during the initial stages of this project. MC would like to thank the CERN Theory Group and the Institute for Theoretical Physics at ETH Zürich for hospitality during the completion of this project. JJ is supported by the project A.AC..FISI.771 PRIN 2010-2011. MC and JS are supported by MINECO, under grant numbers FPA2010-17915 and FPA2013-47836-C3-1/2-P, the FPU program (MC), by the European Commission through the contract PITN-GA-2012316704 (HIGGSTOOLS) and by Junta de Andalucía grants FQM 101 and FQM 6552. GP is supported by the IRG, ISF, the Gruber award, and ERC-2013-CoG grant (TOPCHARM \# 614794). Furthermore, a significant part of this work was done when GP held a Staff position at CERN and JJ was a postdoctoral fellow at the Weizmann Institute. Note added: while this paper was in its final phase of preparations [61] appeared that have some level of overlap with the results presented by us. 


\section{A Model description}

We provide in this appendix a detailed description of the relevant features of the model. Further information in terms of motivation and extra collider implications can be found in the original reference [37]. We assume the first two SM quark generations and the RH bottom quark to be fully elementary and the RH top quark to be fully composite. The third generation SM quark doublet is embedded in a 5 of $\mathrm{SO}(5)$ while the top partners are assumed to live in a 4 of $\mathrm{SO}(4)$. In the basis we are considering these embeddings read

$$
\left(Q_{L}^{5}\right)_{I}=\frac{1}{\sqrt{2}}\left(\begin{array}{c}
\mathrm{i} b_{L} \\
b_{L} \\
\mathrm{i} t_{L} \\
-t_{L} \\
0
\end{array}\right), \quad \Psi^{i}=\frac{1}{\sqrt{2}}\left(\begin{array}{c}
\mathrm{i}\left(B-X_{5 / 3}\right) \\
B+X_{5 / 3} \\
\mathrm{i}\left(T+X_{2 / 3}\right) \\
-T+X_{2 / 3}
\end{array}\right),
$$

where $I=1, \ldots, 5$ and $i=1, \ldots, 4$, respectively.

The Lagrangian involving the fermions and gluons reads, in the elementary-composite basis

$$
\begin{aligned}
\mathcal{L}= & \bar{q}_{L} \mathrm{i} \not D q_{L}+\bar{t}_{R} \mathrm{i} \not D t_{R}+\bar{\Psi} \mathrm{i}(\not D+\mathrm{i} \phi) \Psi-M_{\Psi} \bar{\Psi} \Psi \\
& +\left[\mathrm{i} c_{1}\left(\bar{\Psi}_{R}\right)_{i} \gamma^{\mu} d_{\mu}^{i} t_{R}+y f\left(\bar{Q}_{L}^{5}\right)^{I} U_{I i} \Psi_{R}^{i}+y c_{2} f\left(\bar{Q}_{L}^{5}\right)^{I} U_{I 5} t_{R}+\text { h.c. }\right] \\
& -\frac{1}{2} \operatorname{Tr}\left[G_{\mu \nu}^{e}\right]^{2}-\frac{1}{2} \operatorname{Tr}\left[G_{\mu \nu}^{c}\right]^{2}+\frac{1}{2} M_{c}^{2}\left(G_{\mu}^{c}-\frac{g_{e}}{g_{c}} G_{\mu}^{e}\right)^{2},
\end{aligned}
$$

where the contribution from the Goldstone boson matrix $U$ and the $d$ symbol is given explicitly below. In the Lagrangian above $f$ is the scale characterizing the strong coupling scale while $y$ and $c_{1,2}$ are dimensionless parameters expected to be of order one. The covariant derivatives read explicitly

$$
\begin{aligned}
\mathrm{i} D_{\mu} q_{L} & =\left(\mathrm{i} \partial_{\mu}+g \frac{\sigma^{i}}{2} W_{\mu}^{i}+\frac{g^{\prime}}{6} B_{\mu}+g_{e} G_{\mu}^{e}\right) q_{L}, \\
\mathrm{i} D_{\mu} t_{R} & =\left(\mathrm{i} \partial_{\mu}+\frac{2 g^{\prime}}{3} B_{\mu}+g_{c} G_{\mu}^{c}\right) t_{R}, \\
\mathrm{i} D_{\mu} \Psi & =\left(\mathrm{i} \partial_{\mu}+\frac{2 g^{\prime}}{3} B_{\mu}+g_{c} G_{\mu}^{c}\right) \Psi .
\end{aligned}
$$

Note that the elementary (composite) gluon $G_{\mu}^{e}\left(G_{\mu}^{c}\right)$ couples only to the elementary (composite) quarks with coupling $g_{e}\left(g_{c}\right)$. The mass matrix for the gluons can be diagonalized by means of the following rotation

$$
\left(\begin{array}{c}
G_{\mu}^{e} \\
G_{\mu}^{c}
\end{array}\right)=\left(\begin{array}{cc}
\cos \theta_{3} & -\sin \theta_{3} \\
\sin \theta_{3} & \cos \theta_{3}
\end{array}\right)\left(\begin{array}{c}
g_{\mu} \\
G_{\mu}
\end{array}\right),
$$

with

$$
\tan \theta_{3}=\frac{g_{e}}{g_{c}}
$$


so that we end up with the massless SM gluon, $g_{\mu}$, that couples universally to all the quarks with coupling

$$
g_{s}=g_{c} \sin \theta_{3}=g_{e} \cos \theta_{3},
$$

and a heavy gluon, $G_{\mu}$, with mass

$$
M_{G}=\frac{M_{c}}{\cos \theta_{3}},
$$

and couplings to elementary and composite fields

$$
\begin{aligned}
& g_{e} G_{\mu}^{e}=-g_{e} \sin \theta_{3} G_{\mu}+\ldots=-\frac{g_{s}^{2}}{\sqrt{g_{c}^{2}-g_{s}^{2}}} G_{\mu}+\ldots, \\
& g_{c} G_{\mu}^{c}=g_{c} \cos \theta_{3} G_{\mu}+\ldots=\sqrt{g_{c}^{2}-g_{s}^{2}} G_{\mu}+\ldots
\end{aligned}
$$

respectively.

The remaining terms in the Lagrangian (A.2) read

$$
\begin{aligned}
\mathrm{i} \bar{\Psi}_{R}^{i} d_{i} t_{R}= & \frac{g}{\sqrt{2}} s_{h}\left[\left(\bar{X}_{5 / 3}\right)_{R} W^{+}-\bar{B}_{R} W^{-}\right] t_{R} \\
& -\frac{g}{2 c_{W}} s_{h}\left[\bar{T}_{R}+\left(\bar{X}_{2 / 3}\right)_{R}\right] Z t_{R}+\mathrm{i}\left[\left(\bar{X}_{2 / 3}\right)_{R}-\bar{T}_{R}\right] \frac{\not \rho}{f} t_{R}, \\
\bar{\Psi}\left(\frac{2 g^{\prime}}{3} \not B-\not\right) \Psi= & \frac{g}{c_{W}}\left(-\frac{1}{2}+\frac{s_{W}^{2}}{3}\right) \bar{B} Z B B+\frac{g}{c_{W}}\left(\frac{1}{2}-\frac{5 s_{W}^{2}}{3}\right) \bar{X}_{5 / 3} Z Z X_{5 / 3} \\
& +\frac{g}{c_{W}}\left(\frac{1}{2} c_{h}-\frac{2 s_{W}^{2}}{3}\right) \bar{T} \not Z T+\frac{g}{c_{W}}\left(-\frac{1}{2} c_{h}-\frac{2 s_{W}^{2}}{3}\right) \bar{X}_{2 / 3} Z X_{2 / 3} \\
& +\frac{g}{\sqrt{2}}\left\{\bar{B} W^{-}\left[c_{h / 2}^{2} T+s_{h / 2}^{2} X_{2 / 3}\right]+\bar{X}_{5 / 3} W^{+}\left[s_{h / 2}^{2} T+c_{h / 2}^{2} X_{2 / 3}\right]+\text { h.c. }\right\} \\
& +\operatorname{photon} \operatorname{couplings}, \\
\left(\bar{Q}_{L}^{5}\right)^{I} U_{I i} \Psi_{R}^{i}= & \bar{b}_{L} B_{R}+\bar{t}_{L}\left[c_{h / 2}^{2} T_{R}+s_{h / 2}^{2}\left(X_{2 / 3}\right)_{R}\right], \\
\left(\bar{Q}_{L}^{5}\right)^{I} U_{I 5} t_{R}= & -\frac{1}{\sqrt{2}} s_{h} \bar{t}_{L} t_{R},
\end{aligned}
$$

where we have denoted

$$
s_{x} \equiv \sin \frac{x}{f}, \quad c_{x} \equiv \cos \frac{x}{f},
$$

except for $s_{W}$ and $c_{W}$, which are the sine and cosine of the Weinberg angle. $\rho$ is the physical Higgs boson and $h$ reads, in the unitary gauge

$$
h \equiv\langle h\rangle+\rho,
$$

with

$$
f_{\langle h\rangle}=v \approx 246 \mathrm{GeV} .
$$

Open Access. This article is distributed under the terms of the Creative Commons Attribution License (CC-BY 4.0), which permits any use, distribution and reproduction in any medium, provided the original author(s) and source are credited. 


\section{References}

[1] G. Perez, Best analysis tools, talk given at the 2014 Zurich Phenomenology Workshop, January 8-10, Zurich, Switzerland (2014).

[2] CMS collaboration, Inclusive search for a vector-like $T$ quark with charge $2 / 3$ in pp collisions at $\sqrt{s}=8 \mathrm{TeV}$, Phys. Lett. B 729 (2014) 149 [arXiv:1311.7667] [INSPIRE].

[3] ATLAS collaboration, Search for pair and single production of new heavy quarks that decay to a $Z$ boson and a third-generation quark in pp collisions at $\sqrt{s}=8$ TeV with the ATLAS detector, JHEP 11 (2014) 104 [arXiv:1409.5500] [INSPIRE].

[4] ATLAS collaboration, A search for $t \bar{t}$ resonances in the lepton plus jets final state with ATLAS using $14 \mathrm{fb}^{-1}$ of pp collisions at $\sqrt{s}=8$ TeV, ATLAS-CONF-2013-052 (2013).

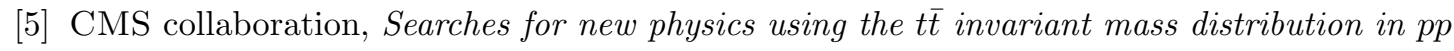
collisions at $\sqrt{s}=8$ TeV, Phys. Rev. Lett. 111 (2013) 211804 [Erratum ibid. 112 (2014) 119903] [arXiv:1309.2030] [INSPIRE].

[6] M.S. Carena, E. Ponton, J. Santiago and C.E.M. Wagner, Electroweak constraints on warped models with custodial symmetry, Phys. Rev. D 76 (2007) 035006 [hep-ph/0701055] [INSPIRE].

[7] C. Anastasiou, E. Furlan and J. Santiago, Realistic composite Higgs models, Phys. Rev. D 79 (2009) 075003 [arXiv:0901.2117] [INSPIRE].

[8] C. Grojean, O. Matsedonskyi and G. Panico, Light top partners and precision physics, JHEP 10 (2013) 160 [arXiv:1306.4655] [INSPIRE].

[9] P. Biancofiore, L. Da Rold, S. Jager, G. Perez and A. Weiler, to appear.

[10] M. Schmaltz, Physics beyond the standard model (theory): introducing the little Higgs, Nucl. Phys. Proc. Suppl. 117 (2003) 40 [hep-ph/0210415] [InSPIRE].

[11] M. Ciuchini, E. Franco, S. Mishima and L. Silvestrini, Electroweak precision observables, new physics and the nature of a 126 GeV Higgs boson, JHEP 08 (2013) 106 [arXiv: 1306.4644] [INSPIRE].

[12] O. Matsedonskyi, G. Panico and A. Wulzer, Light top partners for a light composite Higgs, JHEP 01 (2013) 164 [arXiv:1204.6333] [INSPIRE].

[13] M. Redi and A. Tesi, Implications of a light Higgs in composite models, JHEP 10 (2012) 166 [arXiv:1205.0232] [INSPIRE].

[14] D. Marzocca, M. Serone and J. Shu, General composite Higgs models, JHEP 08 (2012) 013 [arXiv:1205.0770] [INSPIRE].

[15] A. Pomarol and F. Riva, The composite Higgs and light resonance connection, JHEP 08 (2012) 135 [arXiv:1205.6434] [INSPIRE].

[16] G. Panico, M. Redi, A. Tesi and A. Wulzer, On the tuning and the mass of the composite Higgs, JHEP 03 (2013) 051 [arXiv:1210.7114] [INSPIRE].

[17] D. Pappadopulo, A. Thamm and R. Torre, A minimally tuned composite Higgs model from an extra dimension, JHEP 07 (2013) 058 [arXiv: 1303.3062] [INSPIRE].

[18] J. Barnard, T. Gherghetta, A. Medina and T.S. Ray, Radiative corrections to the composite Higgs mass from a gluon partner, JHEP 10 (2013) 055 [arXiv: 1307.4778] [INSPIRE].

[19] A. Carmona and F. Goertz, A naturally light Higgs without light top partners, arXiv: 1410.8555 [INSPIRE]. 
[20] L. Randall and R. Sundrum, A large mass hierarchy from a small extra dimension, Phys. Rev. Lett. 83 (1999) 3370 [hep-ph/9905221] [INSPIRE].

[21] N. Arkani-Hamed, M. Porrati and L. Randall, Holography and phenomenology, JHEP 08 (2001) 017 [hep-th/0012148] [INSPIRE].

[22] K. Agashe, A. Belyaev, T. Krupovnickas, G. Perez and J. Virzi, LHC signals from warped extra dimensions, Phys. Rev. D 77 (2008) 015003 [hep-ph/0612015] [INSPIRE].

[23] B. Lillie, L. Randall and L.-T. Wang, The bulk RS KK-gluon at the LHC, JHEP 09 (2007) 074 [hep-ph/0701166] [INSPIRE].

[24] K. Agashe et al., LHC signals for warped electroweak neutral gauge bosons, Phys. Rev. D 76 (2007) 115015 [arXiv:0709.0007] [INSPIRE].

[25] N. Vignaroli, New W-prime signals at the LHC, Phys. Rev. D 89 (2014) 095027 [arXiv: 1404.5558] [INSPIRE].

[26] M. Carena, A.D. Medina, B. Panes, N.R. Shah and C.E.M. Wagner, Collider phenomenology of gauge-Higgs unification scenarios in warped extra dimensions, Phys. Rev. D 77 (2008) 076003 [arXiv:0712.0095] [inSPIRE].

[27] J. Santiago, The elusive gluon at the LHC, talk given at the Workshop on High Precision LHC Physics, May 23, ETH Zuirch, Switzerland (2014).

[28] J. Santiago, New physics in $t \bar{t}+X$ resonances, talk given at the Topical workshop on top quark differential distributions, September 26-28, Cannes, France (2014).

[29] R. Barcelo, A. Carmona, M. Masip and J. Santiago, Stealth gluons at hadron colliders, Phys. Lett. B 707 (2012) 88 [arXiv:1106.4054] [INSPIRE].

[30] R. Barcelo, A. Carmona, M. Chala, M. Masip and J. Santiago, Single vectorlike quark production at the LHC, Nucl. Phys. B 857 (2012) 172 [arXiv:1110.5914] [inSPIRE].

[31] C. Bini, R. Contino and N. Vignaroli, Heavy-light decay topologies as a new strategy to discover a heavy gluon, JHEP 01 (2012) 157 [arXiv:1110.6058] [INSPIRE].

[32] A. Carmona, M. Chala and J. Santiago, New Higgs production mechanism in composite Higgs models, JHEP 07 (2012) 049 [arXiv: 1205.2378] [INSPIRE].

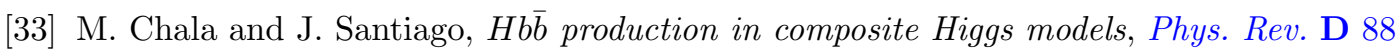
(2013) 035010 [arXiv:1305.1940] [InSPIRE].

[34] K. Agashe, R. Contino and A. Pomarol, The minimal composite Higgs model, Nucl. Phys. B 719 (2005) 165 [hep-ph/0412089] [INSPIRE].

[35] R. Contino, L. Da Rold and A. Pomarol, Light custodians in natural composite Higgs models, Phys. Rev. D 75 (2007) 055014 [hep-ph/0612048] [INSPIRE].

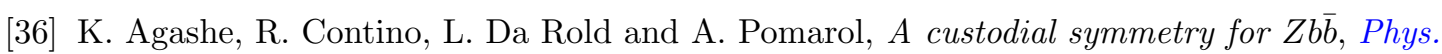
Lett. B 641 (2006) 62 [hep-ph/0605341] [INSPIRE].

[37] A. De Simone, O. Matsedonskyi, R. Rattazzi and A. Wulzer, A first top partner hunter's guide, JHEP 04 (2013) 004 [arXiv: 1211.5663] [INSPIRE].

[38] ATLAS collaboration, M. Petteni, A search for $t \bar{t}$ resonances in the dilepton channel in $1.04 \mathrm{fb}^{-1}$ of pp collisions at $\sqrt{\mathrm{s}}=7 \mathrm{TeV}$ with the ATLAS experiment, arXiv:1111.6933 [INSPIRE]. 
[39] CMS Collaboration, Search for ttbar resonances in dilepton+jets final states in pp collisions at $8 \mathrm{TeV}$, CMS-PAS-B2G-12-007 (2012).

[40] CMS collaboration, Search for anomalous top quark pair production in the boosted all-hadronic final state using pp collisions at $\sqrt{s}=8 \mathrm{TeV}$, CMS-PAS-B2G-12-005 (2012).

[41] ATLAS collaboration, Search for resonances decaying into top-quark pairs using fully hadronic decays in pp collisions with ATLAS at $\sqrt{s}=7$ TeV, JHEP 01 (2013) 116 [arXiv: 1211.2202] [INSPIRE].

[42] CMS collaboration, Search for top-quark partners with charge $5 / 3$ in the same-sign dilepton final state, Phys. Rev. Lett. 112 (2014) 171801 [arXiv:1312.2391] [INSPIRE].

[43] J.A. Aguilar-Saavedra, R. Benbrik, S. Heinemeyer and M. Pérez-Victoria, Handbook of vectorlike quarks: mixing and single production, Phys. Rev. D 88 (2013) 094010 [arXiv: 1306.0572] [INSPIRE].

[44] O. Matsedonskyi, G. Panico and A. Wulzer, On the interpretation of top partners searches, arXiv: 1409.0100 [INSPIRE].

[45] A. Alloul, N.D. Christensen, C. Degrande, C. Duhr and B. Fuks, FeynRules $2.0-A$ complete toolbox for tree-level phenomenology, Comput. Phys. Commun. 185 (2014) 2250 [arXiv: 1310.1921] [INSPIRE].

[46] F. Maltoni and T. Stelzer, MadEvent: automatic event generation with MadGraph, JHEP 02 (2003) 027 [hep-ph/0208156] [INSPIRE].

[47] T. Sjöstrand, S. Mrenna and P.Z. Skands, PYTHIA 6.4 physics and manual, JHEP 05 (2006) 026 [hep-ph/0603175] [INSPIRE].

[48] M.L. Mangano, M. Moretti, F. Piccinini and M. Treccani, Matching matrix elements and shower evolution for top-quark production in hadronic collisions, JHEP 01 (2007) 013 [hep-ph/0611129] [INSPIRE].

[49] P.M. Nadolsky et al., Implications of CTEQ global analysis for collider observables, Phys. Rev. D 78 (2008) 013004 [arXiv:0802.0007] [INSPIRE].

[50] M. Czakon, P. Fiedler and A. Mitov, Total top-quark pair-production cross section at hadron colliders through $O\left(\alpha_{S}^{4}\right)$, Phys. Rev. Lett. 110 (2013) 252004 [arXiv: 1303.6254] [INSPIRE].

[51] M. Cacciari, G.P. Salam and G. Soyez, FastJet user manual, Eur. Phys. J. C 72 (2012) 1896 [arXiv: 1111.6097] [INSPIRE].

[52] M. Cacciari, G.P. Salam and G. Soyez, The anti- $k_{t}$ jet clustering algorithm, JHEP 04 (2008) 063 [arXiv: 0802.1189] [INSPIRE].

[53] ATLAS collaboration, Search for $t \bar{t}$ resonances in the lepton plus jets final state with ATLAS using $4.7 \mathrm{fb}^{-1}$ of pp collisions at $\sqrt{s}=7 \mathrm{TeV}$, Phys. Rev. D 88 (2013) 012004 [arXiv: 1305.2756] [INSPIRE].

[54] D.E. Kaplan, K. Rehermann, M.D. Schwartz and B. Tweedie, Top tagging: a method for identifying boosted hadronically decaying top quarks, Phys. Rev. Lett. 101 (2008) 142001 [arXiv:0806.0848] [INSPIRE].

[55] D. Krohn, J. Thaler and L.-T. Wang, Jet trimming, JHEP 02 (2010) 084 [arXiv:0912.1342] [INSPIRE].

[56] ATLAS collaboration, Studies of sensitivity to new dilepton and ditop resonances with an upgraded ATLAS detector at a high-luminosity LHC, ATL-PHYS-PUB-2013-003 (2013). 
[57] M. Backović, G. Perez, T. Flacke and S.J. Lee, LHC top partner searches beyond the 2 TeV mass region, arXiv: 1409.0409 [INSPIRE].

[58] L.G. Almeida, S.J. Lee, G. Perez, G. Sterman and I. Sung, Template overlap method for massive jets, Phys. Rev. D 82 (2010) 054034 [arXiv:1006.2035] [INSPIRE].

[59] M. Backovic, O. Gabizon, J. Juknevich, G. Perez and Y. Soreq, Measuring boosted tops in semi-leptonic $t \bar{t}$ events for the standard model and beyond, JHEP 04 (2014) 176 [arXiv: 1311.2962] [INSPIRE].

[60] S. Willocq, Prospects on beyond the standard model physics at the HL-LHC, talk given at the ECFA High Luminosity LHC Experiments Workshop, October 21-23, Aix-Le-Bains, France (2014).

[61] D. Greco and D. Liu, Hunting composite vector resonances at the LHC: naturalness facing data, arXiv:1410.2883 [INSPIRE]. 